\title{
Design of orthogonal and biorthogonal lapped transforms satisfying perception related constraints
}

Citation for published version (APA):

Bölcskei, H., Heusdens, R., Theunis, R., \& Janssen, A. J. E. M. (2000). Design of orthogonal and biorthogonal lapped transforms satisfying perception related constraints. IEEE Transactions on Image Processing, 9(5), 760772. https://doi.org/10.1109/83.841513

DOI:

10.1109/83.841513

Document status and date:

Published: 01/01/2000

\section{Document Version:}

Publisher's PDF, also known as Version of Record (includes final page, issue and volume numbers)

\section{Please check the document version of this publication:}

- A submitted manuscript is the version of the article upon submission and before peer-review. There can be important differences between the submitted version and the official published version of record. People interested in the research are advised to contact the author for the final version of the publication, or visit the $\mathrm{DOI}$ to the publisher's website.

- The final author version and the galley proof are versions of the publication after peer review.

- The final published version features the final layout of the paper including the volume, issue and page numbers.

Link to publication

\section{General rights}

Copyright and moral rights for the publications made accessible in the public portal are retained by the authors and/or other copyright owners and it is a condition of accessing publications that users recognise and abide by the legal requirements associated with these rights.

- Users may download and print one copy of any publication from the public portal for the purpose of private study or research.

- You may not further distribute the material or use it for any profit-making activity or commercial gain

- You may freely distribute the URL identifying the publication in the public portal.

If the publication is distributed under the terms of Article 25fa of the Dutch Copyright Act, indicated by the "Taverne" license above, please follow below link for the End User Agreement:

www.tue.nl/taverne

Take down policy

If you believe that this document breaches copyright please contact us at:

openaccess@tue.nl

providing details and we will investigate your claim. 


\title{
Design of Orthogonal and Biorthogonal Lapped Transforms Satisfying Perception Related Constraints
}

\author{
Helmut Bölcskei, Member, IEEE, Richard Heusdens, Rik Theunis, and Augustus J. E. M. Janssen
}

\begin{abstract}
We propose a new efficient method for the design of orthogonal and biorthogonal lapped transforms for image coding applications. It is shown how perception related constraints such as decay and smoothness of the filters' impulse responses can be incorporated in the optimization procedure. A decomposition of lapped transforms (orthogonal and biorthogonal) with $\mathbf{5 0 \%}$ overlap leads to an efficient recursive optimization procedure, which is robust with respect to initial solutions. The importance of this decomposition lies in the fact that it allows to decouple the design of the even-symmetric and the odd-symmetric filters and hence drastically reduces the number of variables to be optimized. It furthermore reveals all the variables predetermined by perception related and coding-efficiency related constraints imposed on the filters. We present design and coding examples demonstrating the perceptual performance and the rate-distortion performance of the resulting transforms.
\end{abstract}

Index Terms-Image coding, transform coding.

\section{INTRODUCTION AND OUTLINE}

$\mathbf{I}$ $\mathrm{T}$ is well known that the discrete cosine transform (DCT) [1], which is used in the JPEG image compression standard, suffers especially at low bit rates from blocking effects. In audio coding these blocking effects are perceived as clicking effects. In order to circumvent this drawback lapped orthogonal transforms (LOT's) have been proposed [2]-[6]. The basic difference between LOT's and block transforms, such as the DCT, is that the LOT basis functions are overlapping and therefore allow "smooth" transitions at the boundaries, which reduces or even eliminates blocking effects.

Although lapped transforms ${ }^{1}$ (LT's) are very popular in image coding, it seems that there are no efficient systematic procedures for the design of perceptually constrained LT's in

\footnotetext{
Manuscript received April 21, 1997; revised September 7, 1999. This work was performed while the first author was on leave at Philips Research Laboratories, Eindhoven, The Netherlands. This work was supported by FWF under Grants P10531-ÖPH and J1629-TEC and by Philips Research Laboratories, Eindhoven. The associate editor coordinating the review of this manuscript and approving it for publication was Dr. Robert Forchheimer.

H. Bölcskei is with the Information Systems Laboratory, Department of Electrical Engineering, Stanford University, Stanford, CA 94305-9510 USA. He is currently on leave from the Department of Communications, Vienna University of Technology, Vienna, Austria.

R. Heusdens is with the Department of Electrical Engineering, Circuits and Systems Group, Delft University of Technology, 2628 CD Delft, The Netherlands.

R. Theunis is with Intreanet Multimedia Engineering, 1991 XD Velserbroek, The Netherlands.

A. J. E. M. Janssen is with Philips Research Laboratories, 5656 AA Eindhoven, The Netherlands.

Publisher Item Identifier S 1057-7149(00)03574-0.

${ }^{1}$ The abbreviation LT stands for both lapped orthogonal and lapped biorthogonal transforms with $50 \%$ overlap.
}

the literature. In this paper, we propose an efficient recursive procedure for the design of LT's ${ }^{2}$ (orthogonal and biorthogonal) with $50 \%$ overlap. We show how perceptually relevant constraints such as decay and smoothness of the filters' impulse responses and coding-efficiency related constraints can be incorporated systematically in the design procedure. Our approach is based on a decomposition of LT's, which allows to decouple the design of the even-symmetric and the odd-symmetric filters (transform basis functions) and hence drastically reduces the number of variables to be optimized. It furthermore reveals all the variables predetermined by the constraints imposed on the filters. In the approach we suggest the even-symmetric filters are designed such that they satisfy perception related and coding-efficiency related constraints and the odd-symmetric filters are derived from the even-symmetric filters by means of a unitary transformation that keeps desirable properties such as frequency selectivity, smoothness in the time domain and so forth. Our decomposition leads to a new efficient recursive optimization procedure, that is fast and robust with respect to initial solutions. We even designed 256-channel LT's with filters of length 512 using the method developed in the paper. In general in image coding applications short filters are desired. However, for audio coding applications it is of interest to consider the design of LT's with long filters. It has been shown in [7] that block transforms and LT's can be interpreted as filter banks (FB's). Since the concepts of FB theory allow an efficient formulation of our design problem, we shall treat the transforms under consideration as perfect reconstruction (PR) FB's. We will furthermore concentrate on one-dimensional transforms.

1) Relation to Other Work: Malvar proposes a recursive method for the design of LOT's with maximum coding gain [6]. The algorithm described in [6] does, however, not take into account perceptually relevant constraints such as smoothness and decay of the filters' impulse responses. Another approach discussed in [6], referred to as quasi-optimal LOT design algorithm, starts from a valid LOT and applies a unitary transformation to the LOT basis functions to obtain a new LOT with specific properties. This algorithm does also not allow to systematically incorporate perception related constraints in the design process. In [8] LOT's with extended overlap have been discussed using a decomposition of polyphase matrices. All discussions in [6], [8] are limited to the orthogonal case. Furthermore, all these methods design both the even-symmetric and the odd-symmetric filters. Hence, they have twice the cost of the methods proposed in this paper. Biorthogonal lapped

\footnotetext{
${ }^{2}$ In the biorthogonal transforms under consideration the synthesis filters have the same length as the analysis filters.
} 


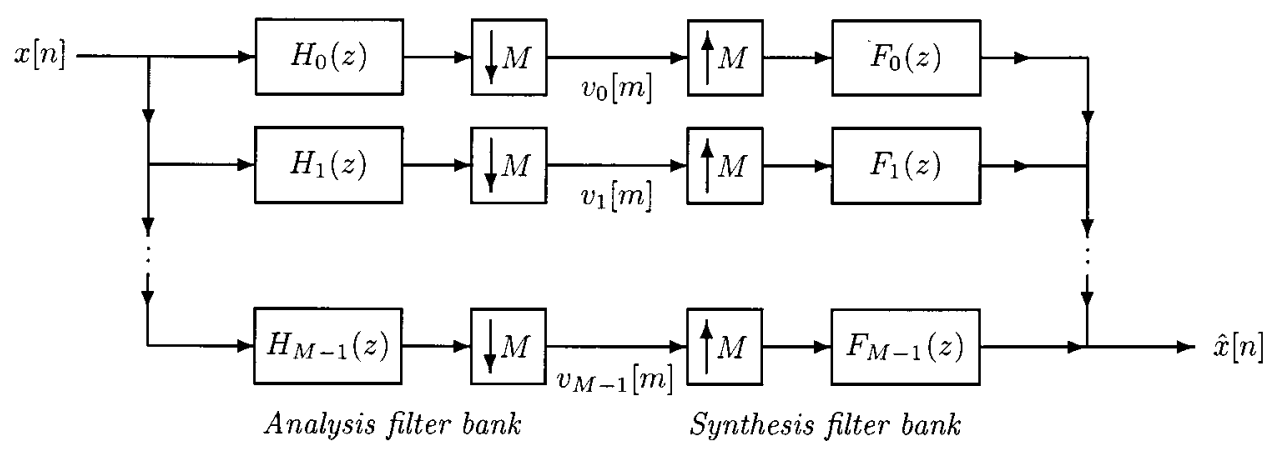

Fig. 1. Critically sampled $M$-channel uniform FB.

transforms (BOLT's) were discussed in [7], [9]. However, no design methods for BOLT's have been reported. BOLT's offer more design flexibility than LOT's, since different restrictions on the analysis and synthesis filters can be imposed.

2) Relation to Previous Own Work: In [10], [11] an ad-hoc method for the design of LOT's has been proposed. This method is based on a decomposition of LOT's with $50 \%$ overlap similar to that described in this paper. In the present paper we extend this decomposition to the biorthogonal case and we introduce a systematic design method for LOT's and BOLT's based on it. We furthermore describe the design of the unitary transformation providing the odd-symmetric filters. Besides that we demonstrate the perceptual performance and the coding performance of the transforms designed using the proposed method.

3) Organization of the Paper: In Section II we briefly review FB's and their relation to LT's and we introduce some notation. In Section III we discuss perception related and codingefficiency related constraints on the filters. We provide an important decomposition of LT's. Based on this decomposition, in Section IV, we formulate a new efficient recursive optimization procedure for orthogonal and biorthogonal LT's. Finally, in Section V we give some design and coding examples demonstrating the perceptual performance and the rate-distortion performance of the resulting filters. Specifically, we demonstrate that the transforms designed using our method achieve the same rate-distortion performance as DCT-based schemes while being substantially superior visually.

Readers familiar with FB's and LT's can proceed to Section III, where the new decomposition of LT's and its consequences and implications are presented. The core part of the paper concerning the optimization is presented in Section IV-A-C.

\section{Filter BANKS AND LAPPed TRANSForms}

In this section, we briefly review critically sampled FB's and their relation to LT's [7], [12], [13].

1) Critically Sampled Filter Banks: We consider an $M$-channel FB [12]-[15] (see Fig. 1) with subsampling by $M$ in each channel, PR and zero delay, so that $\hat{x}[n]=x[n]$ where $x[n]$ and $\hat{x}[n]$ denote the input and reconstructed signal, respectively. The transfer functions of the analysis and synthesis filters are $H_{k}(z)$ and $F_{k}(z)(0 \leq k \leq M-1)$, with corresponding impulse responses $h_{k}[n]$ and $f_{k}[n]$, respectively. In Fig. 1, the $v_{k}[\mathrm{~m}]$ are referred to as subband signals.
2) Polyphase Decomposition: The polyphase decomposition of the analysis filters $H_{k}(z)$ reads [12]-[15]

$$
H_{k}(z)=\sum_{n=0}^{M-1} z^{n} E_{k, n}\left(z^{M}\right), \quad 0 \leq k \leq M-1
$$

where

$$
\begin{aligned}
& E_{k, n}(z)= \sum_{m=-\infty}^{\infty} h_{k}[m M-n] z^{-m}, \\
& 0 \leq k \leq M-1, \quad 0 \leq n \leq M-1
\end{aligned}
$$

is the $n$th polyphase component of the $k$ th analysis filter $H_{k}(z)$. The $M \times M$ analysis polyphase matrix is defined as $[\mathbf{E}(z)]_{k, n}=E_{k, n}(z)[12]-[15]$. The synthesis filters $F_{k}(z)$ can be similarly decomposed

$$
F_{k}(z)=\sum_{n=0}^{M-1} z^{-n} R_{k, n}\left(z^{M}\right), \quad 0 \leq k \leq M-1,
$$

with the synthesis polyphase components

$$
\begin{aligned}
& R_{k, n}(z)= \sum_{m=-\infty}^{\infty} f_{k}[m M+n] z^{-m}, \\
& 0 \leq k \leq M-1, \quad 0 \leq n \leq M-1 .
\end{aligned}
$$

The $M \times M$ synthesis polyphase matrix is defined as $[\mathbf{R}(z)]_{l, k}=R_{k, l}(z)$. A critically sampled FB satisfies the PR condition $\hat{x}[n]=x[n]$ if and only if [12]-[15] $\mathbf{R}(z) \mathbf{E}(z)=\mathbf{E}(z) \mathbf{R}(z)=\mathbf{I}_{M}$, where $\mathbf{I}_{M}$ is the $M \times M$ identity matrix. In the paraunitary case we have ${ }^{3} \tilde{\mathbf{E}}(z) \mathbf{E}(z)=$ $\mathbf{E}(z) \tilde{\mathbf{E}}(z)=\mathbf{I}_{M}$ and hence $\mathbf{R}(z)=\tilde{\mathbf{E}}(z)$. In the biorthogonal case the synthesis polyphase matrix is given by $\mathbf{R}(z)=\mathbf{E}^{-1}(z)$.

Important numerical properties of the FB are determined by its frame bounds [16]-[18] $A$ and $B$. The subband signals $v_{k}[m]$ of a FB satisfy

$$
\begin{aligned}
& A\|x\|^{2} \leq \sum_{k=0}^{M-1} \sum_{m=-\infty}^{\infty}\left|v_{k}[m]\right|^{2} \leq B\|x\|^{2} \\
& \forall x[n] \in l^{2}(\mathbb{Z})
\end{aligned}
$$

with $0<A \leq B<\infty$. In the paraunitary case we have 4 $A=B=1$, whereas in the biorthogonal case, the frame bound ratio $B / A$ determines the "degree of nonorthogonality" of the FB and hence the amount of potential error blow-up due to nonunitarity of the synthesis FB [16]-[18]. A good design

\footnotetext{
${ }^{3}$ The paraconjugate $\tilde{\mathbf{E}}(z)$ of the matrix $\mathbf{E}(z)$ is defined as $\tilde{\mathbf{E}}(z)=$ $\mathbf{E}^{H}\left(1 / z^{*}\right)$ [12], where ${ }^{H}$ stands for conjugate transposition.

${ }^{4}$ Throughout the paper we assume that the $h_{k}[n]$ are normalized, i.e., $\left\|h_{k}\right\|=$ 1 for $k=0,1, \cdots, M-1$.
} 
should aim at $B / A \approx 1$, since $B / A \gg 1$ indicates that the $\mathrm{FB}$ is ill-conditioned in the sense that small perturbations of the subband signals can lead to an error amplification and hence to a big reconstruction error. Therefore, paraunitary FB's have optimum numerical properties. However, for certain applications the constraints imposed on the filters in a paraunitary FB are too restrictive and one has to resort to biorthogonal FB's.

The (tightest possible) frame bounds $A$ and $B$ of a FB are given by the essential infimum and supremum, respectively, of the eigenvalues $\lambda_{n}\left(e^{j 2 \pi \theta}\right)$ of the matrix $\mathbf{E}^{H}\left(e^{j 2 \pi \theta}\right) \mathbf{E}\left(e^{j 2 \pi \theta}\right)$ [16]-[18]:

$$
\begin{aligned}
& A=\operatorname{ess~inf}_{\theta \in[0,1), n=0,1, \cdots, M-1} \lambda_{n}(\theta) \\
& B=\operatorname{ess}_{\sup _{\theta \in[0,1), n=0,1}, \cdots, M-1} \lambda_{n}(\theta) .
\end{aligned}
$$

In general, one has to perform an eigenanalysis of $\mathbf{E}^{H}\left(e^{j 2 \pi \theta}\right) \mathbf{E}\left(e^{j 2 \pi \theta}\right)$ for $\theta \in[0,1)$. We shall see later that for the class of LT's discussed in this paper the matrix $\mathbf{E}^{H}\left(e^{j 2 \pi \theta}\right) \mathbf{E}\left(e^{j 2 \pi \theta}\right)$ is constant, i.e., it does not depend on $\theta$ and therefore the calculation of the frame bounds reduces to the eigenanalysis of a single constant matrix.

3) Filter Banks and Lapped Transforms: FB's provide decompositions into time-shifted versions of a set of basis functions [12], [13]. If the impulse responses have length $2 M$, the FB provides a decomposition into LT's with $50 \%$ overlap [7]. Paraunitary FB's implement decompositions into LOT's [7], [12], [13], whereas biorthogonal FB's provide decompositions into BOLT's [7], [9]. In this paper, we shall consider both LOT's and BOLT's, simply referred to as LT's hereafter.

In the following, we assume that the analysis filters $h_{k}[n](0 \leq k \leq M-1)$ are supported in the interval $-2 M+1 \leq n \leq 0$ and the synthesis filters $f_{k}[n]$ are supported in the interval $0 \leq n \leq 2 M-1$. The analysis polyphase matrix of a FB providing a decomposition into an $\mathrm{LT}$ is given by

$$
\mathbf{E}(z)=\mathbf{E}_{1}+z \mathbf{E}_{2}
$$

where

$$
\mathbf{E}_{1}=\left(\begin{array}{cccc}
h_{0}[0] & h_{0}[-1] & \cdots & h_{0}[-M+1] \\
h_{1}[0] & h_{1}[-1] & \cdots & h_{1}[-M+1] \\
\vdots & \vdots & \vdots & \vdots \\
h_{M-1}[0] & h_{M-1}[-1] & \cdots & h_{M-1}[-M+1]
\end{array}\right)
$$

and

$$
\begin{aligned}
& \mathbf{E}_{2}= \\
& \left(\begin{array}{cccc}
h_{0}[-M] & h_{0}[-M-1] & \cdots & h_{0}[-2 M+1] \\
h_{1}[-M] & h_{1}[-M-1] & \cdots & h_{1}[-2 M+1] \\
\vdots & \vdots & \vdots & \vdots \\
h_{M-1}[-M] & h_{N-1}[-M-1] & \cdots & h_{M-1}[-2 M+1]
\end{array}\right)
\end{aligned}
$$

are $M \times M$ matrices. The synthesis polyphase matrix $\mathbf{R}(z)$ is given by

$$
\mathbf{R}(z)=\mathbf{R}_{1}+z^{-1} \mathbf{R}_{2}
$$

with the $M \times M$ matrices

$$
\mathbf{R}_{1}=\left(\begin{array}{cccc}
f_{0}[0] & f_{1}[0] & \cdots & f_{M-1}[0] \\
f_{0}[1] & f_{1}[1] & \cdots & f_{M-1}[1] \\
\vdots & \vdots & \vdots & \vdots \\
f_{0}[M-1] & f_{1}[M-1] & \cdots & f_{M-1}[M-1]
\end{array}\right)
$$

and

$$
\mathbf{R}_{2}=\left(\begin{array}{cccc}
f_{0}[M] & f_{1}[M] & \cdots & f_{M-1}[M] \\
f_{0}[M+1] & f_{1}[M+1] & \cdots & f_{M-1}[M+1] \\
\vdots & \vdots & \vdots & \vdots \\
f_{0}[2 M-1] & f_{1}[2 M-1] & \cdots & f_{M-1}[2 M-1]
\end{array}\right) .
$$

For PR (with zero delay) it is necessary and sufficient that $\mathbf{R}(z) \mathbf{E}(z)=\mathbf{E}(z) \mathbf{R}(z)=\mathbf{I}_{M}$ [12], [13], which implies

$$
\mathbf{R}_{1} \mathbf{E}_{1}+\mathbf{R}_{2} \mathbf{E}_{2}=\mathbf{E}_{1} \mathbf{R}_{1}+\mathbf{E}_{2} \mathbf{R}_{2}=\mathbf{I}_{M}
$$

and

$$
\mathbf{R}_{1} \mathbf{E}_{2}=\mathbf{R}_{2} \mathbf{E}_{1}=\mathbf{E}_{1} \mathbf{R}_{2}=\mathbf{E}_{2} \mathbf{R}_{1}=\mathbf{0}_{M},
$$

where $\mathbf{0}_{M}$ is the $M \times M$ zero matrix.

\section{ORTHOGONAL AND BIORTHOGONAL LAPPED TRANSFORMS}

In this section, we formulate the constraints on the filters to be designed. The constraints will be grouped into coding-efficiency related constraints and perception related constraints. We present a decomposition of LT's, which allows to formulate an efficient recursive design procedure (see Section IV). The importance of this decomposition lies in the fact that it allows to decouple the design of the even-symmetric and the odd-symmetric filters and hence drastically reduces the number of variables to be optimized. It furthermore reveals all the variables predetermined by the constraints imposed on the filters. The basic idea of our approach is to design the even-symmetric filters such that they satisfy perception related and coding-efficiency related constraints and to derive the odd-symmetric filters from the even-symmetric filters by means of a unitary transformation that keeps desirable properties such as frequency selectivity, smoothness in the time domain and so forth.

\section{A. Constraints on the Filters}

1) Coding-Efficiency Related Constraints: Let us start with the coding-efficiency related constraints. In most practical coding systems the FB channels are coded mutually independent. Therefore, the analysis filters should have sufficient frequency selectivity. In practical coding systems we should aim at designing the analysis filters such that their transfer functions do not overlap too much. It is furthermore desirable that all analysis filters except for the lowpass filter have a zero at DC frequency. This guarantees that DC information can be represented with only one channel, which leads to the most efficient representation of, e. g., flat backgrounds.

2) Perception-Related Constraints: We shall next discuss constraints, which are important from a perceptual point of view. Especially in image coding high frequency channels are often roughly quantized. Since the DC component of images 
should be reconstructed as accurately as possible, it is desirable that all synthesis filters except for the lowpass filter have a zero at DC frequency, since otherwise errors introduced in the high frequency channels will be spread out to the "important" DC component.

Since the contrast-sensitivity function of the human visual system is roughly isotropic (rotation-invariant) [19], [20] and the visual sensitivity of human observers decreases symmetrically at and on both sides of luminance changes [21], the synthesis filters should be linear phase filters.

Long synthesis filters give rise to ringing effects around edges and should be avoided. Therefore, we restrict our attention to LT's with $50 \%$ overlap. Another important requirement is that blocking effects should be eliminated or minimized. This implies that the synthesis filter impulse responses should be "smooth" and decay to zero at the endpoints of their supporting intervals.

\section{B. Decomposition of Lapped Transforms}

In this section, we shall derive a decomposition of LT's (orthogonal and biorthogonal). This decomposition extends a similar decomposition for the orthogonal case [10], [11] and shows that the design of LT's can be decoupled into the design of the even-symmetric and the odd-symmetric filters. Thus, the number of variables to be optimized is drastically reduced. For BOLT's we derive a sufficient condition for the synthesis filters to have the same length as the analysis filters. We furthermore discuss the calculation of frame bounds.

Let us start with the derivation of the decomposition. We consider linear phase real-coefficient filters $h_{k}[n], f_{k}[n](0 \leq k \leq$ $M-1$ ) of length $2 M$ with $M$ even. Thus, the shifted versions of the basis functions are overlapping by $50 \%$ of the total length (LT's with 50\% overlap). The analysis polyphase matrix of the corresponding FB can be written as $\mathbf{E}(z)=\mathbf{E}_{1}+z \mathbf{E}_{2}$, where $\mathbf{E}_{1}$ and $\mathbf{E}_{2}$ are given by (4) and (5), respectively. The synthesis polyphase matrix is $\mathbf{R}(z)=\mathbf{R}_{1}+z^{-1} \mathbf{R}_{2}$, where $\mathbf{R}_{1}$ and $\mathbf{R}_{2}$ are given by (6) and (7), respectively.

It has been shown in [22] that in an $M$-channel critically sampled PR FB the analysis FB has linear phase filters if and only if the synthesis FB has linear phase filters. Furthermore, for $M$ even, there are $M / 2$ symmetric filters and $M / 2$ antisymmetric filters [22]. We thus set $h_{k}[n]=h_{k}[-2 M+1-n]$ for $k=0,1, \cdots, M / 2-1$ and $h_{k}[n]=-h_{k}[-2 M+1-n]$ for $k=M / 2, M / 2+1, \cdots, M-1$. Consequently, $f_{k}[n]=$ $f_{k}[2 M-1-n]$ for $k=0,1, \cdots, M / 2-1$ and $f_{k}[n]=$ $-f_{k}[2 M-1-n]$ for $k=M / 2, M / 2+1, \cdots, M-1$.

We furthermore partition the $M \times M$ matrices $\mathbf{E}_{1}$ and $\mathbf{R}_{1}$ as

$$
\mathbf{E}_{1}=\left[\begin{array}{l}
\mathbf{E}_{11} \\
\mathbf{E}_{21}
\end{array}\right] \quad \mathbf{R}_{1}=\left[\begin{array}{ll}
\mathbf{R}_{11} & \mathbf{R}_{21}
\end{array}\right]
$$

where $\mathbf{E}_{11}$ and $\mathbf{E}_{21}$ are $(M / 2) \times M$ matrices and $\mathbf{R}_{11}$ and $\mathbf{R}_{21}$ are $M \times(M / 2)$ matrices. From the linear phase property of the filters it follows that

$$
\mathbf{E}_{2}=\left[\begin{array}{c}
\mathbf{E}_{11} \mathbf{J}_{M} \\
-\mathbf{E}_{21} \mathbf{J}_{M}
\end{array}\right] \quad \mathbf{R}_{2}=\left[\begin{array}{ll}
\mathbf{J}_{M} \mathbf{R}_{11} & -\mathbf{J}_{M} \mathbf{R}_{21}
\end{array}\right]
$$

where $\mathbf{J}_{M}$ is the $M \times M$ counter-identity matrix given by

$$
\mathbf{J}_{M}=\left(\begin{array}{cccc}
0 & 0 & \cdots & 1 \\
0 & \cdots & 1 & 0 \\
\vdots & \vdots & \vdots & \vdots \\
1 & 0 & \cdots & 0
\end{array}\right) .
$$

Note that postmultiplication by $\mathbf{J}_{M}$ amounts to renumbering the columns in reverse order, whereas premultiplication by $\mathbf{J}_{M}$ corresponds to renumbering the rows in reverse order.

As already stated in the introduction of this section we want to derive the odd-symmetric filters from the even-symmetric filters by means of a transformation. Therefore, the matrices $\mathbf{E}_{21}$ and $\mathbf{R}_{21}$ containing the coefficients of the odd-symmetric filters are written as $\mathbf{E}_{21}=\mathbf{B}_{a} \mathbf{E}_{11}$ and $\mathbf{R}_{21}=\mathbf{R}_{11} \mathbf{B}_{a}^{-1}$ with some nonsingular $(M / 2) \times(M / 2)$ matrix $\mathbf{B}_{a}$. We shall next provide a sufficient condition on the matrix $\mathbf{B}_{a}$ guaranteeing that the synthesis FB is FIR and that the synthesis filters have the same length as the analysis filters.

Proposition 3.1: Let $\mathbf{E}(z)$ be given by

$$
\mathbf{E}(z)=\left[\begin{array}{c}
\mathbf{E}_{11} \\
\mathbf{B}_{a} \mathbf{E}_{11}
\end{array}\right]+z\left[\begin{array}{c}
\mathbf{E}_{11} \mathbf{J}_{M} \\
-\mathbf{B}_{a} \mathbf{E}_{11} \mathbf{J}_{M}
\end{array}\right] \text {. }
$$

For $\mathbf{B}_{a}$ unitary, ${ }^{5}$ i.e., $\mathbf{B}_{a} \mathbf{B}_{a}^{T}=\mathbf{B}_{a}^{T} \mathbf{B}_{a}=\mathbf{I}_{M / 2}$ the synthesis FB is FIR and the synthesis filters have the same length as the analysis filters. The corresponding synthesis polyphase matrix $\mathbf{R}(z)=\mathbf{E}^{-1}(z)$ is of the form ${ }^{6}$

$$
\mathbf{R}(z)=\left[\begin{array}{lll}
\mathbf{R}_{11} & \mathbf{R}_{11} \mathbf{B}_{a}^{T}
\end{array}\right]+z^{-1}\left[\mathbf{J}_{M} \mathbf{R}_{11} \quad-\mathbf{J}_{M} \mathbf{R}_{11} \mathbf{B}_{a}^{T}\right]
$$

with

$$
\mathbf{R}_{11}=\frac{1}{2}\left(\mathbf{E}_{11}^{T} \mathbf{E}_{11}+\mathbf{J}_{M} \mathbf{E}_{11}^{T} \mathbf{E}_{11} \mathbf{J}_{M}\right)^{-1} \mathbf{E}_{11}^{T} .
$$

Proof: According to [12]-[15] det $\mathbf{E}(z)=c z^{-K}$ with $c \in \mathbb{R}$ and $K \in \mathbb{Z}$ is necessary and sufficient for the PR synthesis FB to be FIR if the analysis FB is FIR. The condition det $\mathbf{E}(z)=c z^{-K}$ is equivalent to $\operatorname{det}[\tilde{\mathbf{E}}(z) \mathbf{E}(z)]=c^{2}$. Indeed, it can easily be seen that for $\mathbf{B}_{a}$ unitary

$$
\operatorname{det}[\tilde{\mathbf{E}}(z) \mathbf{E}(z)]=2 \operatorname{det}\left(\mathbf{E}_{11}^{T} \mathbf{E}_{11}+\mathbf{J}_{M} \mathbf{E}_{11}^{T} \mathbf{E}_{11} \mathbf{J}_{M}\right)
$$

which, of course, is a constant independent of $z$. This shows that for $\mathbf{B}_{a}$ unitary the synthesis FB is FIR. Furthermore, $\mathbf{R}(z)=$ $\mathbf{E}^{-1}(z)=[\tilde{\mathbf{E}}(z) \mathbf{E}(z)]^{-1} \tilde{\mathbf{E}}(z)$, which using

$$
\begin{aligned}
& \left(\mathbf{E}_{11}^{T} \mathbf{E}_{11}+\mathbf{J}_{M} \mathbf{E}_{11}^{T} \mathbf{E}_{11} \mathbf{J}_{M}\right)^{-1} \mathbf{J}_{M} \\
& \quad=\mathbf{J}_{M}\left(\mathbf{E}_{11}^{T} \mathbf{E}_{11}+\mathbf{J}_{M} \mathbf{E}_{11}^{T} \mathbf{E}_{11} \mathbf{J}_{M}\right)^{-1}
\end{aligned}
$$

for $\mathbf{B}_{a}$ unitary gives

$$
\mathbf{R}(z)=\left[\begin{array}{ll}
\mathbf{R}_{11} & \mathbf{R}_{11} \mathbf{B}_{a}^{T}
\end{array}\right]+z^{-1}\left[\mathbf{J}_{M} \mathbf{R}_{11} \quad-\mathbf{J}_{M} \mathbf{R}_{11} \mathbf{B}_{a}^{T}\right]
$$

with

$$
\mathbf{R}_{11}=\frac{1}{2}\left(\mathbf{E}_{11}^{T} \mathbf{E}_{11}+\mathbf{J}_{M} \mathbf{E}_{11}^{T} \mathbf{E}_{11} \mathbf{J}_{M}\right)^{-1} \mathbf{E}_{11}^{T} .
$$

Thus, the synthesis filters have the same length as the analysis filters.

Note that in the orthogonal case the synthesis filters automatically have the same length as the analysis filters. In the biorthog-

\footnotetext{
${ }^{5}$ Since we are considering real-coefficient filters, the entries in $\mathbf{B}_{a}$ are realvalued.

${ }^{6}$ Note that for $\mathbf{B}_{a}$ unitary, we have $\mathbf{B}_{a}^{-1}=\mathbf{B}_{a}^{T}$.
} 
onal case, however, this will in general not be true. Proposition 3.1 shows that $\mathbf{B}_{a}$ unitary is sufficient for the synthesis FB to be FIR with synthesis filters having the same length as the analysis filters. In the following, we shall always assume that $\mathbf{B}_{a}$ is unitary. Therefore, the transformation providing the transition from the even-symmetric filters to the odd-symmetric filters is a unitary transformation. We note that Proposition 3.1 is consistent with the "orthogonality of overlapping blocks" condition [7] $\mathbf{E}_{1}^{T} \mathbf{E}_{2}=\mathbf{0}_{M}$, which is sufficient for the synthesis filters to be FIR with the same length as the analysis filters. It is easily seen that for $\mathbf{B}_{a}$ unitary $\mathbf{E}_{1}^{T} \mathbf{E}_{2}=\mathbf{0}_{M}$. From Proposition 3.1 we furthermore conclude that the design of LT's can be reduced to the design of the matrices $\mathbf{E}_{11}, \mathbf{R}_{11}$ (or equivalently the even-symmetric filters) and the unitary matrix $\mathbf{B}_{a}$. Fixing the analysis $\mathrm{FB}$, i.e., the matrix $\mathbf{E}_{11}$ and calculating the synthesis FB according to (12) will not guarantee that the synthesis FB has the desired properties (zeros at DC frequency, frequency selectivity, smoothness and decay of the filters). In the paraunitary (orthogonal) case we have to design the matrices $\mathbf{E}_{11}$ and $\mathbf{B}_{a}$.

The decompositions (11) and (12) allow to focus on the design of the filters with even symmetry or equivalently the matrices $\mathbf{E}_{11}$ and $\mathbf{R}_{11}$. Once these filters are designed we calculate the matrix $\mathbf{B}_{a}$ (see Section III-D) such that the odd-symmetric filters inherit desirable properties from the even-symmetric filters. We have thus split up the design into two stages, which drastically reduces the number of variables that have to be optimized at a time.

1) PR Conditions: In the following, we shall always assume that $\mathbf{E}_{21}=\mathbf{B}_{a} \mathbf{E}_{11}$ and $\mathbf{R}_{21}=\mathbf{R}_{11} \mathbf{B}_{a}^{-1}$. The PR condition $\mathbf{R}(z) \mathbf{E}(z)=\mathbf{I}_{M}$ can be rephrased in terms of the matrices $\mathbf{R}_{11}$ and $\mathbf{E}_{11}$ as

$$
\mathbf{R}_{11} \mathbf{E}_{11}+\mathbf{J}_{M} \mathbf{R}_{11} \mathbf{E}_{11} \mathbf{J}_{M}=\frac{1}{2} \mathbf{I}_{M}
$$

or equivalently using $\mathbf{R}(z) \mathbf{E}(z)=\mathbf{E}(z) \mathbf{R}(z)=\mathbf{I}_{M}$ we have

$$
\mathbf{E}_{11} \mathbf{R}_{11}=\frac{1}{2} \mathbf{I}_{M / 2} \quad \mathbf{E}_{11} \mathbf{J}_{M} \mathbf{R}_{11}=\mathbf{0}_{M / 2} .
$$

2) Paraunitarity Conditions: The FB is paraunitary, i.e., $\tilde{\mathbf{E}}(z) \mathbf{E}(z)=\mathbf{I}_{M}$, if and only if

$$
\mathbf{E}_{11}^{T} \mathbf{E}_{11}+\mathbf{J}_{M} \mathbf{E}_{11}^{T} \mathbf{E}_{11} \mathbf{J}_{M}=\frac{1}{2} \mathbf{I}_{M}
$$

or equivalently using $\tilde{\mathbf{E}}(z) \mathbf{E}(z)=\mathbf{E}(z) \tilde{\mathbf{E}}(z)=\mathbf{I}_{M}$, we get

$$
\mathbf{E}_{11} \mathbf{E}_{11}^{T}=\frac{1}{2} \mathbf{I}_{M / 2} \quad \mathbf{E}_{11} \mathbf{J}_{M} \mathbf{E}_{11}^{T}=\mathbf{0}_{M / 2} .
$$

Note that for a paraunitary FB $\mathbf{R}_{11}=\mathbf{E}_{11}^{T}$.

3) Frame Bounds: The frame bounds $A, B$ are obtained as the infimum and the supremum, of the eigenvalues of the matrix $\mathbf{E}^{H}\left(e^{j 2 \pi \theta}\right) \mathbf{E}\left(e^{j 2 \pi \theta}\right)$. We shall next show that in our case $\mathbf{E}^{H}\left(e^{j 2 \pi \theta}\right) \mathbf{E}\left(e^{j 2 \pi \theta}\right)$ is independent of $\theta$ and therefore the calculation of the frame bounds reduces to an eigenanalysis of a single constant matrix. We have

$$
\mathbf{E}^{H}\left(e^{j 2 \pi \theta}\right) \mathbf{E}\left(e^{j 2 \pi \theta}\right)=2\left[\mathbf{E}_{11}^{T} \mathbf{E}_{11}+\mathbf{J}_{M} \mathbf{E}_{11}^{T} \mathbf{E}_{11} \mathbf{J}_{M}\right] .
$$

Therefore

$$
\begin{aligned}
& A=\min _{i=0,1, \cdots, M-1} \lambda_{i} \\
& B=\max _{i=0,1, \cdots, M-1} \lambda_{i}
\end{aligned}
$$

where $\lambda_{i}(i=0,1, \cdots, M-1)$ denotes the eigenvalues of $2\left[\mathbf{E}_{11}^{T} \mathbf{E}_{11}+\mathbf{J}_{M} \mathbf{E}_{11}^{T} \mathbf{E}_{11} \mathbf{J}_{M}\right]$.

\section{Decomposition into Symmetric and Antisymmetric Parts}

In this section, we further decompose the LT's under consideration by expressing the matrices $\mathbf{E}_{11}$ and $\mathbf{R}_{11}$ in terms of their symmetric and antisymmetric parts. This decomposition reveals the variables that are predetermined due to the constraints on the zeros of the analysis and the synthesis filters at $z=1$ (see Proposition 3.2). We have

$$
\begin{aligned}
& \mathbf{E}_{e}=\frac{1}{2}\left(\mathbf{E}_{11}+\mathbf{E}_{11} \mathbf{J}_{M}\right) \\
& \mathbf{E}_{o}=\frac{1}{2}\left(\mathbf{E}_{11}-\mathbf{E}_{11} \mathbf{J}_{M}\right) \text { and } \\
& \mathbf{R}_{e}=\frac{1}{2}\left(\mathbf{R}_{11}+\mathbf{J}_{M} \mathbf{R}_{11}\right) \\
& \mathbf{R}_{o}=\frac{1}{2}\left(\mathbf{R}_{11}-\mathbf{J}_{M} \mathbf{R}_{11}\right)
\end{aligned}
$$

where $\mathbf{E}_{e}\left(\mathbf{E}_{o}\right)$ and $\mathbf{R}_{e}\left(\mathbf{R}_{o}\right)$ denotes the even(odd)-symmetric part of the matrix $\mathbf{E}_{11}\left(\mathbf{R}_{11}\right)$. The following symmetry properties will be used subsequently:

$$
\begin{aligned}
& \mathbf{E}_{e} \mathbf{J}_{M}=\mathbf{E}_{e}, \quad \mathbf{E}_{o} \mathbf{J}_{M}=-\mathbf{E}_{o}, \\
& \mathbf{J}_{M} \mathbf{R}_{e}=\mathbf{R}_{e} \quad \text { and } \quad \mathbf{J}_{M} \mathbf{R}_{o}=-\mathbf{R}_{o} .
\end{aligned}
$$

We furthermore have $\mathbf{E}_{11}=\mathbf{E}_{e}+\mathbf{E}_{o}$ and $\mathbf{R}_{11}=\mathbf{R}_{e}+\mathbf{R}_{\circ}$. Equations (11) and (12) can now be rewritten as

$$
\mathbf{E}(z)=\left[\begin{array}{c}
\mathbf{E}_{e}+\mathbf{E}_{o} \\
\mathbf{B}_{a}\left(\mathbf{E}_{e}+\mathbf{E}_{o}\right)
\end{array}\right]+z\left[\begin{array}{c}
\mathbf{E}_{e}-\mathbf{E}_{o} \\
-\mathbf{B}_{a}\left(\mathbf{E}_{e}-\mathbf{E}_{o}\right)
\end{array}\right]
$$

and

$$
\begin{aligned}
& \mathbf{R}(z)=\left[\begin{array}{ll}
\mathbf{R}_{e}+\mathbf{R}_{o} & \left(\mathbf{R}_{e}+\mathbf{R}_{o}\right) \mathbf{B}_{a}^{T}
\end{array}\right] \\
& +z^{-1}\left[\mathbf{R}_{e}-\mathbf{R}_{o} \quad-\left(\mathbf{R}_{e}-\mathbf{R}_{o}\right) \mathbf{B}_{a}^{T}\right] .
\end{aligned}
$$

We note that such a decomposition already appears in [6] for the special case of a LOT designed from a DCT. In [6] $\mathbf{E}_{e}$ and $\mathbf{E}_{o}$ are the even-symmetric and odd-symmetric DCT basis functions, respectively. We will demonstrate subsequently that the decompositions (18) and (19) are of importance in a more general context.

1) PR Conditions: Using $\mathbf{E}_{11}=\mathbf{E}_{e}+\mathbf{E}_{o}$ and $\mathbf{R}_{11}=\mathbf{R}_{e}+$ $\mathbf{R}_{o}$ in (14) it follows that the FB is PR if and only if

$$
\mathbf{R}_{e} \mathbf{E}_{e}+\mathbf{R}_{o} \mathbf{E}_{o}=\frac{1}{4} \mathbf{I}_{M}
$$

or equivalently using (15) we get

$$
\mathbf{E}_{e} \mathbf{R}_{e}=\mathbf{E}_{o} \mathbf{R}_{o}=\frac{1}{4} \mathbf{I}_{M / 2} .
$$

From (13) it follows that

$$
\begin{aligned}
& \mathbf{R}_{e}=\frac{1}{4}\left(\mathbf{E}_{e}^{T} \mathbf{E}_{e}+\mathbf{E}_{o}^{T} \mathbf{E}_{o}\right)^{-1} \mathbf{E}_{e}^{T} \\
& \mathbf{R}_{o}=\frac{1}{4}\left(\mathbf{E}_{e}^{T} \mathbf{E}_{e}+\mathbf{E}_{o}^{T} \mathbf{E}_{o}\right)^{-1} \mathbf{E}_{o}^{T} .
\end{aligned}
$$

2) Paraunitarity Conditions: It can be shown that the FB is paraunitary (the LT is an LOT) if and only if

$$
\begin{aligned}
\mathbf{E}_{e}^{T} \mathbf{E}_{e}+\mathbf{E}_{o}^{T} \mathbf{E}_{o} & =\frac{1}{4} \mathbf{I}_{M} \quad \text { or equivalently } \\
\mathbf{E}_{e} \mathbf{E}_{e}^{T} & =\mathbf{E}_{o} \mathbf{E}_{o}^{T}=\frac{1}{4} \mathbf{I}_{M / 2} .
\end{aligned}
$$


The frame bounds $A$ and $B$ of the FB are given by the minimum and the maximum, respectively, of the eigenvalues of the matrix $4\left(\mathbf{E}_{e}^{T} \mathbf{E}_{e}+\mathbf{E}_{o}^{T} \mathbf{E}_{o}\right)$.

Proposition 3.2: In an LT providing PR the analysis filters $H_{k}(z)(k=1,2, \cdots, M-1)$ and the synthesis filters $F_{k}(z)(k=1,2, \cdots, M-1)$ have a zero at $z=1$ if and only if ${ }^{7}$

$$
\begin{array}{ll}
\mathbf{E}_{e}(0, n)=\alpha, & n=0,1, \cdots, M-1 \\
\mathbf{R}_{e}(k, 0)=\beta, & k=0,1, \cdots, M-1
\end{array}
$$

with $\alpha \beta=(1 / 4 M)$.

Proof: For $H_{k}(1)=F_{k}(1)=0$ with $k=1,2, \cdots, M-1$ we must have $\sum_{n=0}^{M-1} \mathbf{E}_{e}(k, n)=0$ for $k=1,2, \cdots, M-1$ and $\sum_{k=0}^{M-1} \mathbf{R}_{e}(k, n)=0$ for $n=1,2, \cdots, M-1$. We shall first prove sufficiency of (24) and (25). Define the $M \times M$ matrices $\mathbf{U}^{T}=\left[\begin{array}{ll}\mathbf{E}_{e}^{T} & \mathbf{E}_{o}^{T}\end{array}\right]$ and $\mathbf{S}=\left[\begin{array}{ll}\mathbf{R}_{e} & \mathbf{R}_{o}\end{array}\right]$. Using (20), we get

$$
\mathbf{U S}=\left[\begin{array}{ll}
\mathbf{E}_{e} \mathbf{R}_{e} & \mathbf{E}_{e} \mathbf{R}_{o} \\
\mathbf{E}_{o} \mathbf{R}_{e} & \mathbf{E}_{o} \mathbf{R}_{o}
\end{array}\right]=\frac{1}{4} \mathbf{I}_{M} .
$$

This implies that $\mathbf{S U}=\frac{1}{4} \mathbf{I}_{M}$. Equations (24) and (25) can be rewritten as $\alpha \mathbf{s}=\mathbf{U}^{T} \mathbf{e}_{1}$ and $\beta \mathbf{s}=\mathbf{S e}_{1}$, where $\mathbf{s}=$ $\left[\begin{array}{llll}1 & 1 & \cdots & 1\end{array}\right]^{T}$ is an $M \times 1$ vector with all ones and $\mathbf{e}_{1}=$ $\left[\begin{array}{llll}1 & 0 & \cdots & 0\end{array}\right]^{T}$ is an $M \times 1$ vector. Multiplying $\beta \mathbf{s}=\mathbf{S e}_{1}$ by $\mathbf{U}$ from the left and using $\mathbf{U S}=\frac{1}{4} \mathbf{I}_{M}$ yields $\mathbf{U s}=(1 / 4 \beta) \mathbf{e}_{1}$, which implies that $H_{k}(1)=0$ for $k=1,2, \cdots, M-1$. Proceeding the same way starting from $\alpha \mathbf{s}=\mathbf{U}^{T} \mathbf{e}_{1}$ and $\mathbf{S}^{T} \mathbf{U}^{T}=$ $\frac{1}{4} \mathbf{I}_{M}$ it follows that $F_{k}(1)=0$ for $k=1,2, \cdots, M-1$. The necessity of (24) and (25) is shown as follows. Let us assume that $H_{k}(1)=F_{k}(1)=0$ with $k=1,2, \cdots, M-1$. Hence $\sum_{n=0}^{M-1} \mathbf{E}_{e}(k, n)=0(k=1,2, \cdots, M-1)$, which implies that $\mathbf{U s}_{\mathbf{s}}=\alpha^{\prime} \mathbf{e}_{1}$ with some constant $\alpha^{\prime}$. Thus $\mathbf{S U s}=\alpha^{\prime} \mathbf{S e}_{1}$ and using $\mathbf{S U}=\frac{1}{4} \mathbf{I}_{M}$ we get $\mathbf{s}=4 \alpha^{\prime} \mathbf{S e}_{1}$, which implies that the first column of $\boldsymbol{R}_{e}$ is constant. Setting $\beta=\left(1 / 4 \alpha^{\prime}\right)$ (25) is shown. Starting from $F_{k}(1)=0(k=1,2, \cdots, M-1)$ or equivalently $\mathbf{S}^{T} \mathbf{S}=\beta^{\prime} \mathbf{e}_{1}$ it can be shown similarly, that $\mathbf{S}=$ $4 \beta^{\prime} \mathbf{U}^{T} \mathbf{e}_{1}$, which implies that the first row of $\mathbf{E}_{e}$ is constant. Setting $\alpha=\left(1 / 4 \beta^{\prime}\right)(24)$ is proved. Finally, from $\mathbf{E}_{e} \mathbf{R}_{e}=\frac{1}{4} \mathbf{I}_{M / 2}$ it follows that $\alpha \beta=(1 / 4 M)$, which concludes the proof.

In the paraunitary case, where $\mathbf{R}_{e}=\mathbf{E}_{e}^{T}$ (24) with $\alpha=$ $(1 / 2 \sqrt{M})$ is necessary and sufficient for all filters except for the lowpass filter to have a zero at DC frequency. Note that the odd-symmetric filters have a zero at $z=1$ by definition.

\section{Design of the Unitary Transformation}

In this section, we show how the unitary matrix $\mathbf{B}_{a}$ can be designed. We assume that the even-symmetric filters are designed such that they satisfy the constraints discussed in Section III-A.

One way to obtain the odd-symmetric filters is to modulate the even-symmetric filters, which means that for an even-symmetric filter $h_{k}[n]$ the corresponding odd-symmetric filter $h_{M-k-1}[n]$ satisfies

$$
\begin{aligned}
H_{M-k-1}\left(e^{j 2 \pi \theta}\right) & =H_{k}\left(e^{j 2 \pi(\theta-(1 / 2))}\right) \quad \text { so that } \\
h_{M-k-1}[n] & =(-1)^{n} h_{k}[n] .
\end{aligned}
$$

Recall that the filters $h_{k}[n]$ with $k=0,1, \cdots, M / 2-1$ are even-symmetric and the filters $h_{k}[n]$ with $k=M / 2$,

${ }^{7} \mathbf{E}_{e}(k, n)$ denotes the $n$th element in the $k$ th row of $\mathbf{E}_{e}$.
$M / 2+1, \cdots, M-1$ are odd-symmetric. Let $\mathbf{D}_{M}=$ $\operatorname{diag}\left\{(-1)^{n}\right\}_{n=0}^{M-1}$. Then (26) can be written as

$$
\mathbf{E}_{21}=\mathbf{J}_{M / 2} \mathbf{E}_{11} \mathrm{D} \text {. }
$$

This choice of $\mathbf{E}_{21}$ together with $\mathbf{R}_{21}=\mathbf{D R}_{11} \mathbf{J}_{M / 2}$ combined with (14) or the equivalent conditions (15) will in general not yield a PR LT, since the conditions (9) are not automatically satisfied. One could, however, impose additional restrictions on $\mathbf{E}_{11}$ and $\mathbf{R}_{11}$ such that the resulting transform has PR. However, this amounts to imposing additional side constraints on the even-symmetric filters. Clearly, the quality of the resulting filters (frequency selectivity and so forth) will not be as good as that of the filters obtained using our transformation approach.

Since shifting the even-symmetric filters in frequency to obtain the odd-symmetric filters will guarantee that the odd-symmetric filters keep desirable properties of the even-symmetric filters, such as frequency selectivity, smoothness of the impulse responses and so forth, we shall try to approximate $\mathbf{E}_{21}=$ $\mathbf{J}_{M / 2} \mathbf{E}_{11} \mathbf{D}$ by the matrix $\mathbf{B}_{a} \mathbf{E}_{11}$ with $\mathbf{B}_{a}$ unitary. A good approximation to $\mathbf{E}_{21}=\mathbf{J}_{M / 2} \mathbf{E}_{11} \mathbf{D}$ will guarantee that the oddsymmetric filters are approximately frequency-shifted versions of the even-symmetric filters and thus share desirable properties of the even-symmetric filters. As we shall see later (see the design examples in Section $\mathrm{V}$ ) it is relatively easy to find a unitary matrix $\mathbf{B}_{a}$ that provides a good approximation.

In general the row space of $\mathbf{B}_{a} \mathbf{E}_{11}$ will not coincide with the row space of $\mathbf{J}_{M / 2} \mathbf{E}_{11} \mathbf{D}_{M}$. In the case that the two row spaces do not coincide we will approximate $\mathbf{J}_{M / 2} \mathbf{E}_{11} \mathbf{D}_{M}$ in a least-squares sense, i.e., we will approximate $\mathbf{J}_{M / 2} \mathbf{E}_{11} \mathbf{D}_{M}$ by the orthogonal projection of the row space of $\mathbf{J}_{M / 2} \mathbf{E}_{11} \mathbf{D}_{M}$ onto the row space of $\mathbf{B}_{a} \mathbf{E}_{11}$. This means that

$$
\begin{aligned}
& \mathbf{B}_{a} \mathbf{E}_{11}=\mathbf{J}_{M / 2} \mathbf{E}_{11} \mathbf{D}_{M}\left(\mathbf{B}_{a} \mathbf{E}_{11}\right)^{T}\left(\mathbf{B}_{a} \mathbf{E}_{11}\left(\mathbf{B}_{a} \mathbf{E}_{11}\right)^{T}\right)^{-1} \\
& \mathbf{B}_{a} \mathbf{E}_{11}=\mathbf{J}_{M / 2} \mathbf{E}_{11} \mathbf{D}_{M} \mathbf{E}_{11}^{T}\left(\mathbf{E}_{11} \mathbf{E}_{11}^{T}\right)^{-1} \mathbf{E}_{11}
\end{aligned}
$$

so that $\mathbf{B}_{a}$ is given by

$$
\mathbf{B}_{a}=\mathbf{J}_{M / 2} \mathbf{E}_{11} \mathbf{D}_{M} \mathbf{E}_{11}^{T}\left(\mathbf{E}_{11} \mathbf{E}_{11}^{T}\right)^{-1} .
$$

Since $\mathbf{J}_{M / 2}, \mathbf{D}_{M}$ and $\mathbf{E}_{11}$ are of full rank (full rank of $\mathbf{E}_{11}$ follows from the PR property of the FB [10], [11]) we conclude that $\mathbf{B}_{a}$ is nonsingular, as required.

In order to have $\mathbf{B}_{a}$ unitary as required, we can apply a GramSchmidt orthogonalization to either the rows or the columns of $\mathbf{B}_{a}$. For perceptual reasons, however, it is advantageous to apply the orthogonalization procedure to the rows of $\mathbf{B}_{a}$. This can be understood as follows. The Gram-Schmidt process says that if $\mathbf{W}=\left\{\mathbf{w}_{n}\right\}_{n=1}^{N}$ is a linearly independent set of vectors, then there exists an orthogonal set $\mathbf{V}=\left\{\mathbf{v}_{n}\right\}_{n=1}^{N}$ such that $\mathbf{v}_{n} \neq \mathbf{0}(n=1,2, \cdots, N)$ and $\mathbf{v}_{n}$ is a linear combination of $\mathbf{w}_{1}, \cdots, \mathbf{w}_{n}$ for $n=1,2, \cdots, N$. The $\mathbf{v}_{n}$ are constructed by

$$
\mathbf{v}_{n}=\mathbf{w}_{n}-\sum_{k=1}^{n-1} \frac{\left\langle\mathbf{w}_{n}, \mathbf{v}_{k}\right\rangle}{\left\|\mathbf{v}_{k}\right\|^{2}} \mathbf{v}_{k} .
$$

Clearly, the idea of the Gram-Schmidt process is to subtract from every new vector its components in the directions that are already settled and normalize it. As a consequence, the deviation of the orthogonalized vectors $\mathbf{v}_{n}$ with respect to the original nonprocessed vectors $\mathrm{w}_{n}$ increases with increasing index, that 
is, $\left\|\mathbf{w}_{n+1}-\mathbf{v}_{n+1}\right\| \geq\left\|\mathbf{w}_{n}-\mathbf{v}_{n}\right\|$ for $n=1,2, \cdots, N-1$. Next consider the construction of $\mathbf{E}_{21}=\mathbf{B}_{a} \mathbf{E}_{11}$ and assume that we have ordered the even-symmetric analysis filters in $\mathbf{E}(z)$ such that the pass-band center-frequency of the frequency responses increases with increasing row index $k$. Obviously, the $k$ th row of $\mathbf{E}_{21}$ is only affected by $\mathbf{E}_{11}$ and the $k$ th row of $\mathbf{B}_{a}$. Since the sensitivity of the human visual system varies as a function of spatial frequency in the sense that it is less sensitive to higher spatial frequencies than to lower spatial frequencies [19], [20], we conclude that errors, i.e., deviations from the ideal $\mathbf{B}_{a}$ given by (28) in the higher order rows of $\mathbf{B}_{a}$ are less critical than errors in the lower order rows of $\mathbf{B}_{a}$. This is so, since errors in the higher order rows of $\mathbf{B}_{a}$ will affect higher frequency filters. Thus the Gram-Schmidt procedure should be applied to the rows rather than to the columns of $\mathbf{B}_{a}$, starting with the first row, then the second one and so forth. We note that if the Gram-Schmidt process is applied to the columns of the matrix $\mathbf{B}_{a}$, the errors, i.e., the deviations from the ideal $\mathbf{B}_{a}$ lead to an error that is uniformly distributed among all filters, which of course is not desirable.

We finally show under what conditions on the matrices $\mathbf{E}_{e}$, $\mathbf{E}_{o}$ and $\mathbf{B}_{a}$ the equation $\mathbf{B}_{a} \mathbf{E}_{11}=\mathbf{J}_{M / 2} \mathbf{E}_{11} \mathbf{D}_{M}$ can be satisfied exactly.

Proposition 3.3: The equation $\mathbf{B}_{a} \mathbf{E}_{11}=\mathbf{J}_{M / 2} \mathbf{E}_{11} \mathbf{D}_{M}$ is satisfied if and only if $\mathbf{B}_{a} \mathbf{E}_{o}=\mathbf{J}_{M / 2} \mathbf{E}_{e} \mathbf{D}_{M}$ and $\mathbf{B}_{a}=\mathbf{J}_{M / 2} \mathbf{B}_{a}^{T} \mathbf{J}_{M / 2}$.

Proof: Assume that $\mathbf{B}_{a} \mathbf{E}_{11}=\mathbf{J}_{M / 2} \mathbf{E}_{11} \mathbf{D}_{M}$. We then have $\mathbf{B}_{a}\left(\mathbf{E}_{e}+\mathbf{E}_{o}\right)=\mathbf{J}_{M / 2}\left(\mathbf{E}_{e}+\mathbf{E}_{o}\right) \mathbf{D}_{M}$. Noting that $\mathbf{J}_{M} \mathbf{D}_{M} \mathbf{J}_{M}=-\mathbf{D}_{M}$ it follows from $\mathbf{E}_{e} \mathbf{J}_{M}=\mathbf{E}_{e}$ that $\mathbf{E}_{e} \mathbf{D}_{M} \mathbf{J}_{M}=\left(\mathbf{E}_{e} \mathbf{J}_{M}\right)\left(\mathbf{J}_{M} \mathbf{D}_{M} \mathbf{J}_{M}\right)=-\mathbf{E}_{e} \mathbf{J}_{M} \mathbf{D}_{M}$ $=-\mathbf{E}_{e} \mathbf{D}_{M}$, which shows that $\mathbf{E}_{e} \mathbf{D}_{M}$ is odd-symmetric. Similarly, it can be shown that $\mathbf{E}_{o} \mathbf{D}_{M}$ is even-symmetric. Therefore, since both $\mathbf{E}_{e}$ and $\mathbf{E}_{o}$ are of full rank, we conclude that $\mathbf{B}_{a} \mathbf{E}_{o}=\mathbf{J}_{M / 2} \mathbf{E}_{e} \mathbf{D}_{M}$ and $\mathbf{B}_{a} \mathbf{E}_{e}=\mathbf{J}_{M / 2} \mathbf{E}_{o} \mathbf{D}_{M}$. Multiplying $\mathbf{B}_{a} \mathbf{E}_{o}=\mathbf{J}_{M / 2} \mathbf{E}_{e} \mathbf{D}_{M}$ from the left with $\mathbf{B}_{a}^{T}$ and using the unitarity of $\mathbf{B}_{a}$ it follows that $\mathbf{E}_{o}=\mathbf{B}_{a}^{T} \mathbf{J}_{M / 2} \mathbf{E}_{e} \mathbf{D}_{M}$, which implies that $\mathbf{B}_{a} \mathbf{E}_{e}=\mathbf{J}_{M / 2} \mathbf{E}_{o} \mathbf{D}_{M}=\mathbf{J}_{M / 2} \mathbf{B}_{a}^{T} \mathbf{J}_{M / 2} \mathbf{E}_{e}$ and hence $\mathbf{B}_{a}=\mathbf{J}_{M / 2} \mathbf{B}_{a}^{T} \mathbf{J}_{M / 2}$.

Conversely, assume that $\mathbf{B}_{a} \mathbf{E}_{o}=\mathbf{J}_{M / 2} \mathbf{E}_{e} \mathbf{D}_{M}$ and $\mathbf{B}_{a}=\mathbf{J}_{M / 2} \mathbf{B}_{a}^{T} \mathbf{J}_{M / 2}$. We then have $\mathbf{J}_{M / 2} \mathbf{E}_{o} \mathbf{D}_{M}=$ $\mathbf{J}_{M / 2} \mathbf{B}_{a}^{T} \mathbf{J}_{M / 2} \mathbf{E}_{e}=\mathbf{B}_{a} \mathbf{E}_{e}$. Hence, $\mathbf{J}_{M / 2} \mathbf{E}_{11} \mathbf{D}_{M}=$ $\mathbf{J}_{M / 2}\left(\mathbf{E}_{e}+\mathbf{E}_{o}\right) \mathbf{D}_{M}=\mathbf{J}_{M / 2} \mathbf{E}_{e} \mathbf{D}_{M}+\mathbf{J}_{M / 2} \mathbf{E}_{o} \mathbf{D}_{M}=$ $\mathbf{B}_{a} \mathbf{E}_{o}+\mathbf{B}_{a} \mathbf{E}_{e}=\mathbf{B}_{a} \mathbf{E}_{11}$, as required.

\section{RECURSIVE OPTIMIZATION OF LAPPED TRANSFORMS}

In this section, we formulate a recursive optimization procedure for LT's. We concentrate on the design of the even-symmetric filters, since as explained in Section III-D the odd-symmetric filters can be obtained from the even-symmetric filters by means of a unitary transformation. We furthermore assume that in the frequency domain the even-symmetric filters and the odd-symmetric filters are alternating. This means that the first filter (corresponding to center frequency 0 ) is even-symmetric, the second filter (corresponding to center frequency $3 /(4 M)$ ) is odd-symmetric and so forth. The design procedure can equivalently be formulated for a different ordering of the filters.

\section{A. Cost Function}

In this subsection we discuss the choice of the cost function in the paraunitary case. We assume that the filters are normalized such that $\left\|h_{k}\right\|=1$ for $k=0,1, \cdots, M / 2-1$. According to the discussion in Section III-A the filters' transfer functions should not overlap too much. We provide two formulations of the cost function that achieve this goal. The first formulation aims at minimizing

$$
J_{1}=\sum_{k=0}^{M / 2-1} E_{s_{k}}
$$

where

$E_{s_{k}}=\int_{0}^{k / M}\left|H_{k}\left(e^{j 2 \pi \theta}\right)\right|^{2} d \theta+\int_{(2 k+1) /(2 M)}^{1 / 2}\left|H_{k}\left(e^{j 2 \pi \theta}\right)\right|^{2} d \theta$

is the energy of the $k$ th analysis filter in its stopband region $0 \leq \theta \leq k / M$ and $(2 k+1) /(2 M) \leq \theta \leq 1 / 2$. Minimizing $J_{1}$ corresponds to maximizing the energy of the $k$ th analysis filter in a frequency band around $(4 k+1) /(4 M)$ with bandwidth $1 /(2 M)$ under the side constraint of constant energy of $h_{k}[n]$. We have

$$
\begin{aligned}
J_{1}= & \sum_{k=0}^{M / 2-1}\left[\int_{0}^{k / M}\left|H_{k}\left(e^{j 2 \pi \theta}\right)\right|^{2} d \theta\right. \\
& \left.+\int_{(2 k+1) /(2 M)}^{1 / 2}\left|H_{k}\left(e^{j 2 \pi \theta}\right)\right|^{2} d \theta\right]
\end{aligned}
$$

which after straightforward manipulation can be written as

$$
J_{1}=\sum_{k=0}^{M / 2-1} \mathbf{h}_{k}^{T} \mathbf{A}_{k} \mathbf{h}_{k}
$$

where the $\mathbf{A}_{k}$ are $2 M \times 2 M$ matrices with elements

$$
\begin{aligned}
\mathbf{A}_{k}(m, n) & \frac{\sin \left(\frac{2 \pi k}{M}(m-n)\right)-\sin \left(\frac{\pi(2 k+1)}{M}(m-n)\right)}{2 \pi(m-n)}
\end{aligned}
$$

for $m \neq n$. For $m=n$ we have $\mathbf{A}_{k}(m, m)=\frac{1}{2}(1-(1 / M))$. We have thus expressed $J_{1}$ as a quadratic form in terms of the filter coefficients $\mathbf{h}_{k}$ and the matrices $\mathbf{A}_{k}$. Note that the expression for $J_{1}$ can be further simplified by taking into account the linear phase property of the filters. Doing so, we get

$$
J_{1}=\sum_{k=0}^{M / 2-1} \tilde{\mathbf{h}}_{k}^{T} \mathbf{S}_{k} \tilde{\mathbf{h}}_{k}
$$

where $\tilde{\mathbf{h}}_{k}^{T}=\left[h_{k}[0] h_{k}[-1] \cdots h_{k}[-M+1]\right]$ and $\mathbf{S}_{k}=\mathbf{A}_{k_{0,0}}+$ $\mathbf{A}_{k_{0,1}} \mathbf{J}_{M}+\mathbf{J}_{M} \mathbf{A}_{k_{1,0}}+\mathbf{J}_{M} \mathbf{A}_{k_{1,1}} \mathbf{J}_{M}$. Here we have written the $2 M \times 2 M$ matrix $\mathbf{A}_{k}$ in terms of the $M \times M$ matrices $\mathbf{A}_{k_{j, l}}$ $(j, l=0,1)$ as

$$
\mathbf{A}_{k}=\left(\begin{array}{cc}
\mathbf{A}_{k_{0}, 0} & \mathbf{A}_{k_{0,1}} \\
\mathbf{A}_{k_{1,0}} & \mathbf{A}_{k_{1,1}}
\end{array}\right)
$$

This formulation of the cost function guarantees a certain degree of overall smoothness of the filters' impulse responses since it aims at minimizing the stopband energy of the filters. However, it does not perform a weighting in the stopband of the filters. Therefore, it is still possible that the resulting filters have significant frequency components far off the center frequency and 


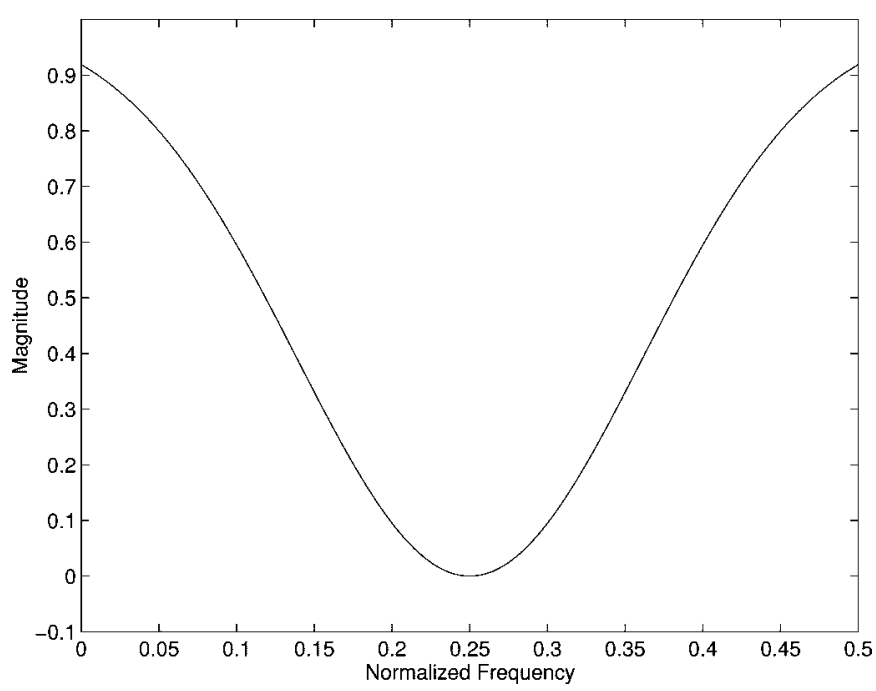

Fig. 2. Typical choice for the weighting function $W_{k}\left(e^{j 2 \pi \theta}\right)$.

thus the filters will not be smooth in the time domain. As we shall see in Section V, the resulting filters will still exhibit "discontinuities" at their boundaries and therefore lead to perceptually annoying artifacts, which are, however, far less annoying than in the DCT case. Forcing the boundary values of the filters' impulse responses to zero (or to a small value) and concurrently minimizing $J_{1}$ will in general lead to discontinuities in the interior of the filters' impulse responses. Incorporating the boundary values of the $h_{k}[n]$ in the cost function and trying to minimize these values leads to "smooth" filters that still exhibit some blocking at the boundaries.

We shall next present an alternative formulation of the cost function. This formulation leads to filters with improved "smoothness" properties and allows to completely eliminate blocking. The cost function is now defined as

$$
J_{2}=\sum_{k=0}^{M / 2-1} \int_{0}^{1 / 2}\left|H_{k}\left(e^{j 2 \pi \theta}\right)\right|^{2} W_{k}\left(e^{j 2 \pi \theta}\right) d \theta
$$

where $W_{k}\left(e^{j 2 \pi \theta}\right)$ is a 1-periodic weighting function that takes into account the frequency localization of $H_{k}\left(e^{j 2 \pi \theta}\right)$. Since our filters are real-valued it suffices to specify $W_{k}\left(e^{j 2 \pi \theta}\right)$ on the interval $0 \leq \theta<0.5$. A typical choice for $W_{k}\left(e^{j 2 \pi \theta}\right)$ is $\left.W_{k}\left(e^{j 2 \pi \theta}\right)=\overline{(\theta}-\theta_{k}\right)^{2}$, where $\theta_{k}=(4 k+1) /(4 M)$. Other possible choices for $W_{k}\left(e^{j 2 \pi \theta}\right)$ are $W_{k}\left(e^{j 2 \pi \theta}\right)=1-G_{k}\left(e^{j 2 \pi \theta}\right)$, where $G_{k}\left(e^{j 2 \pi \theta}\right)$ may be a normalized Gaussian or a normalized raised cosine centered at $\theta_{k}$. In Fig. 2 the weighting function $W_{k}\left(e^{j 2 \pi \theta}\right)$ is depicted for $G_{k}\left(e^{j 2 \pi \theta}\right)$ a normalized Gaussian.

For $W_{k}\left(e^{j 2 \pi \theta}\right)=\left(\theta-\theta_{k}\right)^{2}$ the cost function can be expressed as

$$
J_{2}=\sum_{k=0}^{M / 2-1} \mathbf{h}_{k}^{T} \mathbf{T}_{k} \mathbf{h}_{k}
$$

where the $\mathbf{T}_{k}$ are $2 M \times 2 M$ matrices with elements

$$
\mathbf{T}_{k}(m, n)=\frac{(-1)^{(m-n)}}{(2 \pi(m-n))^{2}}+2 \theta_{k} \frac{1-(-1)^{(m-n)}}{(2 \pi(m-n))^{2}}
$$

for $m \neq n$. For $m=n$ we have $\mathbf{T}_{k}(m, m)=\left(\left(1 / 2-\theta_{k}\right)^{3} / 3\right)+$ $\left(\theta_{k}^{3} / 3\right)$. Taking into account the linear phase property of the filters we get

$$
J_{2}=\sum_{k=0}^{M / 2-1} \tilde{\mathbf{h}}_{k}^{T} \mathbf{U}_{k} \tilde{\mathbf{h}}_{k}
$$

where $\mathbf{U}_{k}=\mathbf{T}_{k_{0,0}}+\mathbf{T}_{k_{0,1}} \mathbf{J}_{M}+\mathbf{J}_{M} \mathbf{T}_{k_{1,0}}+\mathbf{J}_{M} \mathbf{T}_{k_{1,1}} \mathbf{J}_{M}$. Here we have written the $2 M \times 2 M$ matrix $\mathbf{T}_{k}$ in terms of the $M \times M$ matrices $\mathbf{T}_{k_{j, l}}(j, l=0,1)$ as

$$
\mathbf{T}_{k}=\left(\begin{array}{ll}
\mathbf{T}_{k_{0,0}} & \mathbf{T}_{k_{0,1}} \\
\mathbf{T}_{k_{1,0}} & \mathbf{T}_{k_{1,1}}
\end{array}\right)
$$

Minimizing $J_{2}$ under the constraints $h_{k}[0]=0(k=0$, $1, \cdots, M / 2-1$ ) leads to "smooth" filters that do not give rise to blocking effects at the boundaries. Note that forcing the boundary value to be zero combined with the smoothness requirement achieved by the choice of the cost function $J_{2}$ guarantees that the filters smoothly decay to zero at the boundaries. However, these filters will have less frequency selectivity than those obtained by minimizing $J_{1}$ (see the design examples in Section V). Choosing ${ }^{8} W_{k}\left(e^{j 2 \pi \theta}\right)=$ $I_{[0,(k / M)]}+I_{[(2 k+1) /(2 M),(1 / 2)]}$ is easily seen to result in $J_{1}=J_{2}$. Thus $J_{1}$ corresponds to a sharp cutoff in the frequency domain, whereas $J_{2}$ corresponds to a smooth cutoff. Using the cost function $J_{2}$ guarantees overall smoothness of the filter impulse responses, since $J_{2}$ aims at frequency localization of the filters. Good localization in the frequency domain guarantees smoothness in the time domain. Note that $J_{1}$ also aims at good frequency localization. However, since $J_{1}$ does not include a weighting in the stopband region of the filters, it may happen that there are significant frequency components far off the center frequency of the filter and thus the filter will not be smooth in the time domain.

We note that the approach described above is very flexible because it allows to trade off frequency selectivity of the filters for blocking and smoothness by a proper choice of the weighting function $W_{k}\left(e^{j 2 \pi \theta}\right)$.

\section{B. Recursive Optimization in the Orthogonal Case}

In this subsection we introduce a recursive design procedure, by which we mean that we are optimizing one filter after the other. Such a procedure clearly has advantages over a direct design, because the number of variables that have to be optimized simultaneously is drastically reduced, which results in better convergence properties. Another advantage of this approach is that it allows for the design of long filters, which are necessary e.g. in speech and audio coding applications. We formulate the optimization in terms of the matrices $\mathbf{E}_{e}$ and $\mathbf{E}_{o}$. Note that a formulation of the optimization procedure in terms of the matrix $\mathbf{E}_{11}$ does not reveal that due to the side constraint $H_{k}(1)=0$ for $k=1,2, \cdots, M / 2-1$ some of the values in $\mathbf{E}_{11}$ are predetermined. In the approach we suggest this is reflected by the fact that the first row of $\mathbf{E}_{e}$ is constant, i.e., $\mathbf{E}_{e}(0, n)=(1 / 2 \sqrt{M})$. Thus, the optimization of the first basis function reduces to the optimization of the first row of $\mathbf{E}_{\odot}$. The optimization of the other

${ }^{8} I_{[x, y]}$ denotes the indicator function on the interval $[x, y]$. 
basis functions requires a simultaneous optimization of both the corresponding rows in $\mathbf{E}_{e}$ and $\mathbf{E}_{o}$. The side constraints arising from the paraunitarity of the FB are given by (23). The algorithm is as follows.

- Step 1) Find $\mathbf{E}_{o}(1, \cdot)$ that minimizes

$$
J_{1,0}=\tilde{\mathbf{h}}_{0}^{T} \mathbf{S}_{0} \tilde{\mathbf{h}}_{0}+\lambda_{0} h_{0}^{2}[0], \quad \text { or } \quad J_{2,0}=\tilde{\mathbf{h}}_{0}^{T} \mathbf{U}_{0} \tilde{\mathbf{h}}_{0}
$$

where $\lambda_{0}$ is a proper weighting factor, which can be chosen to be zero. The side constraints are given by

$-h_{0}[0]=0$ (eliminate blocking). This side constraint

is to be used in combination with $J_{2,0}$.

$-\left\langle\mathbf{E}_{o}(0, \cdot), \mathbf{E}_{o}(0, \cdot)\right\rangle=1 / 4$ (Normalization).

- Step 2) Set the basis function index to $k=1$.

- Step 3) Find $\mathbf{E}_{e}(k, \cdot)$ and $\mathbf{E}_{o}(k, \cdot)$ that minimize

$$
J_{1, k}=\tilde{\mathbf{h}}_{k}^{T} \mathbf{S}_{k} \tilde{\mathbf{h}}_{k}+\lambda_{k} h_{k}^{2}[0] \quad \text { or } \quad J_{2, k}=\tilde{\mathbf{h}}_{k}^{T} \mathbf{U}_{k} \tilde{\mathbf{h}}_{k}
$$

where $\lambda_{k}$ is a proper weighting factor, which can be chosen to be zero. The side constraints are given by

$-h_{k}[0]=0$ (eliminate blocking). This side constraint is to be used in combination with $J_{2, k}$.

$-\left\langle\mathbf{E}_{e}(k, \cdot), \mathbf{E}_{e}(l, \cdot)\right\rangle=0$ for $l=0,1, \cdots, k-1$ (orthogonality of rows of $\mathbf{E}_{e}$ ).

$-\left\langle\mathbf{E}_{o}(k, \cdot), \mathbf{E}_{o}(l, \cdot)\right\rangle=0$ for $l=0,1, \cdots, k-1$ (orthogonality of rows of $\left.\mathbf{E}_{o}\right)$.

$-\left\langle\mathbf{E}_{e}(k, \cdot), \mathbf{E}_{e}(k, \cdot)\right\rangle=\left\langle\mathbf{E}_{o}(k, \cdot), \mathbf{E}_{o}(k, \cdot)\right\rangle=1 / 4$ (normalized analysis filters, i.e., $\left\|h_{k}\right\|=1$ ).

- Step 4) $k \rightarrow k+1$ (next basis function).

- Step 5) Stop if $k=M / 2-1$; otherwise go back to Step 3.

Choosing $\lambda_{0}$ in Step 1 or $\lambda_{k}$ in Step 3 not equal to zero amounts to including the boundary value of the corresponding impulse response in the cost function. Another possible choice for the cost function results from including $h_{k}[0]$ in the cost function $J_{2, k}$. Then, clearly $h_{k}[0]=0$ must not be a side constraint any more. It is easily seen that there is no need to include the condition on the zeros of the analysis filters at $z=1$, since this is already guaranteed by having a constant first row of $\mathbf{E}_{e}$ (see Proposition 3.2). We note that this recursive approach does not guarantee that the optimization yields the global optimum.

\section{Recursive Optimization in the Biorthogonal Case}

In the biorthogonal case a simultaneous optimization of the analysis and the synthesis FB has to be performed, which means that we are optimizing the first analysis filter and the first synthesis filter concurrently and so on. Designing the analysis FB and calculating the corresponding synthesis FB does not guarantee that the synthesis filters have any of the desired properties except for linear phase.

From (13), it follows that there is a unique relation between the analysis and the synthesis filters. So, one possible approach to the design of a biorthogonal LT would be to optimize $\mathbf{E}_{11}$ (or equivalently $\mathbf{E}_{e}$ and $\mathbf{E}_{o}$ ) and to impose restrictions on the synthesis filters determined by $\mathbf{R}_{11}=\frac{1}{2}\left(\mathbf{E}_{11}^{T} \mathbf{E}_{11}+\mathbf{J}_{M} \mathbf{E}_{11}^{T} \mathbf{E}_{11} \mathbf{J}_{M}\right)^{-1} \mathbf{E}_{11}^{T}$. This approach does, however, not allow for a recursive formulation since in order to compute one of the synthesis filters according to (13) all analysis filters have to be known. Since, a recursive design procedure clearly has advantages over a direct one, we simultaneously optimize the rows in $\mathbf{E}_{e}$ and $\mathbf{E}_{o}$ and the corresponding columns in $\mathbf{R}_{e}$ and $\mathbf{R}_{o}$. The cost function used in the biorthogonal case includes the frequency selectivity of all filters (analysis and synthesis) and "smoothness" of the synthesis filters' impulse responses. Note that there is no need for the analysis filters to decay to zero in the time domain. Thus as we shall see in Section V in the biorthogonal case the analysis filters will have better frequency selectivity than their orthogonal counterparts. The optimization is formulated in terms of the matrices $\mathbf{E}_{e}, \mathbf{E}_{o}, \mathbf{R}_{e}$ and $\mathbf{R}_{o}$. Due to Proposition 3.2 the first row of $\mathbf{E}_{e}$ and the first column of $\mathbf{R}_{e}$ are predetermined. We set $\mathbf{E}_{e}(0, n)=(1 / 2 \sqrt{M})$ and $\mathbf{R}_{e}(k, 0)=(1 / 2 \sqrt{M})$. Thus the optimization of the first analysis filter and the first synthesis filter reduces to the optimization of the first row of $\mathbf{E}_{o}$ and the first column of $\mathbf{R}_{o}$. The algorithm is as follows.

- Step 1) Find $\mathbf{E}_{0}(1, \cdot)$ and $\mathbf{R}_{o}(\cdot, 1)$ that minimize

$$
J_{1,0}=\tilde{\mathbf{h}}_{0}^{T} \mathbf{S}_{0} \tilde{\mathbf{h}}_{0}+\lambda_{1} \tilde{\mathbf{f}}_{0}^{T} \mathbf{S}_{0} \tilde{\mathbf{f}}_{0}+\lambda_{2} f_{0}^{2}[0]
$$

or

$$
J_{2,0}=\tilde{\mathbf{h}}_{0}^{T} \mathbf{S}_{0} \tilde{\mathbf{h}}_{0}+\lambda^{\prime}{ }_{1} \tilde{\mathbf{f}}_{0}^{T} \mathbf{U}_{0} \tilde{\mathbf{f}}_{0}
$$

where $\lambda_{1}, \lambda_{2}$ and $\lambda_{1}^{\prime}$ are proper weighting factors and $\tilde{\mathbf{f}}_{k}=\left[f_{k}[0] f_{k}[1] \cdots f_{k}[M-1]\right]^{T}$. The side constraints are given by

$-f_{0}[0]=0$ (eliminate blocking). This side constraint is to be used in combination with $J_{2,0}$.

$-\left\langle\mathbf{E}_{o}(0, \cdot), \mathbf{E}_{o}(0, \cdot)\right\rangle=1 / 4$ (normalized analysis filters, i.e., $\left.\left\|h_{k}\right\|=1\right)$

$-\left\langle\mathbf{E}_{o}(0, \cdot), \mathbf{R}_{o}(\cdot, 0)\right\rangle=1 / 4$.

- Step 2) Set the basis function index to $k=1$.

- Step 3) Find $\mathbf{E}_{e}(k, \cdot), \mathbf{E}_{o}(k, \cdot), \mathbf{R}_{e}(\cdot, k), \mathbf{R}_{o}(\cdot, k)$ that minimize

$$
J_{1, k}=\tilde{\mathbf{h}}_{k}^{T} \mathbf{S}_{k} \tilde{\mathbf{h}}_{k}+\lambda_{1} \tilde{\mathbf{f}}_{k}^{T} \mathbf{S}_{k} \tilde{\mathbf{f}}_{k}+\lambda_{2} f_{k}^{2}[0]
$$

or

$$
J_{2, k}=\tilde{\mathbf{h}}_{k}^{T} \mathbf{S}_{k} \tilde{\mathbf{h}}_{k}+\lambda_{1}^{\prime} \tilde{\mathbf{f}}_{k}^{T} \mathbf{U}_{k} \tilde{\mathbf{f}}_{k}
$$

where $\lambda_{1}, \lambda_{2}$ and $\lambda_{1}^{\prime}$ are proper weighting factors. The side constraints are given by

$-f_{k}[0]=0$ (eliminate blocking). This side constraint

is to be used in combination with $J_{2, k}$.

$-\left\langle\mathbf{E}_{e}(k, \cdot), \mathbf{R}_{e}(\cdot, l)\right\rangle=\left\langle\mathbf{E}_{o}(k, \cdot), \mathbf{R}_{o}(\cdot, l)\right\rangle=0$ for

$l=0,1, \cdots, k-1$ (biorthogonality)

$-\left\langle\mathbf{E}_{e}(l, \cdot), \mathbf{R}_{e}(\cdot, k)\right\rangle=\left\langle\mathbf{E}_{o}(l, \cdot), \mathbf{R}_{o}(\cdot, k)\right\rangle=0$ for

$l=0,1, \cdots, k-1$ (biorthogonality)

$-\left\langle\mathbf{E}_{e}(k, \cdot), \mathbf{R}_{e}(\cdot, k)\right\rangle=\left\langle\mathbf{E}_{o}(k, \cdot), \mathbf{R}_{o}(\cdot, k)\right\rangle=1 / 4$

(biorthogonality)

$-\left\langle\mathbf{E}_{e}(k, \cdot), \mathbf{E}_{e}(k, \cdot)\right\rangle=\left\langle\mathbf{E}_{o}(k, \cdot), \mathbf{E}_{o}(k, \cdot)\right\rangle=1 / 4$

$\left(\left\|h_{k}\right\|=1\right.$ for $\left.k=0,1, \cdots, M / 2-1\right)$.

- Step 4) $k \rightarrow k+1$ (next basis function).

- Step 5) Stop if $k=M / 2-1$; otherwise go back to Step 3.

As in the orthogonal case, another possible choice for the cost function is obtained by including the boundary value $f_{k}[0]$ in $J_{2, k}$. Then, clearly $f_{k}[0]=0$ must not be a side constraint any more. Using this cost function often results in improved convergence properties.

The advantage of biorthogonal transforms over orthogonal transforms is increased design flexibility. This is mainly due to the fact that we can impose different restrictions on the analysis 

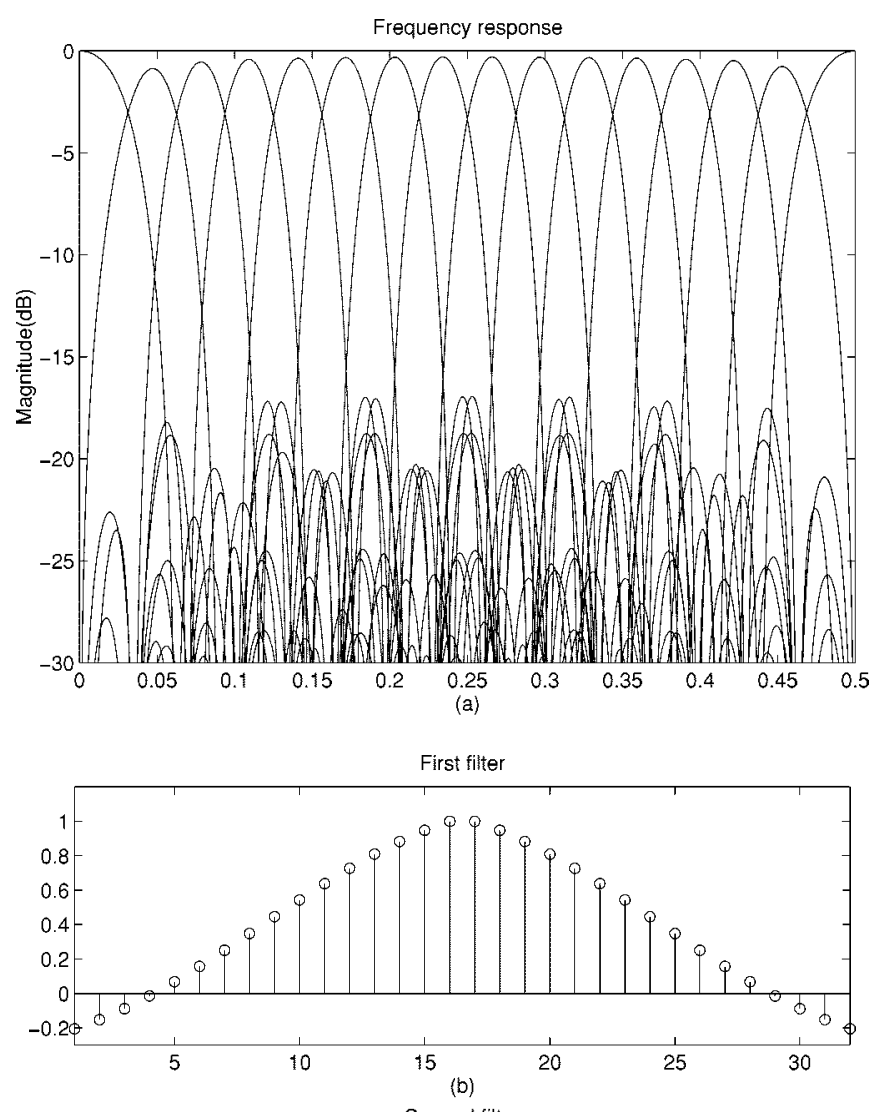

Second filter

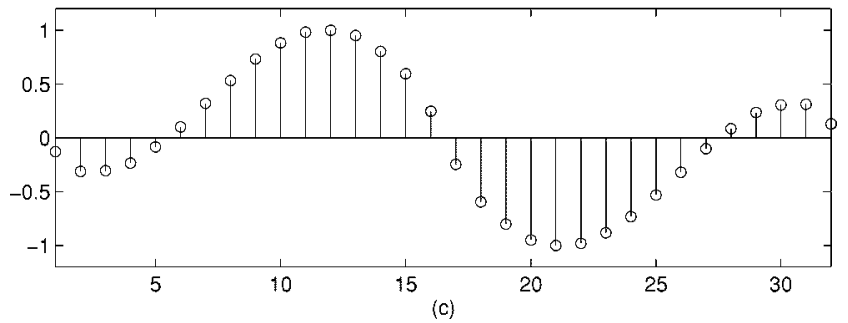

Fig. 3. Sixteen-channel LOT: (a) transfer functions, (b) first filter, and (c) second filter.

and the synthesis filters. However, in hardware implementations orthogonal transforms are preferred because of the saving of silicon area, which is due to the fact that the analysis filters are equal to the synthesis filters (up to time reversal and conjugation). We note that the frame bound ratio $B / A$ determining important numerical properties of the BOLT can be incorporated in the cost function. However, this requires an eigenanalysis of an $M \times M$ matrix in each step of the iteration, which is computationally expensive. Thus, it has to be checked after the design whether the ratio $B / A$ is close to 1 . We found that this is usually the case for the design procedures described in this paper.

\section{Comments}

We conclude this section with some general remarks. The proposed algorithms have been implemented in MATLAB. We designed up to 256-channel LT's with filters of length 512. Our algorithms were found to have excellent convergence properties. They are furthermore very robust with respect to initial solutions. Typically, one can choose a random point as the initial solution.
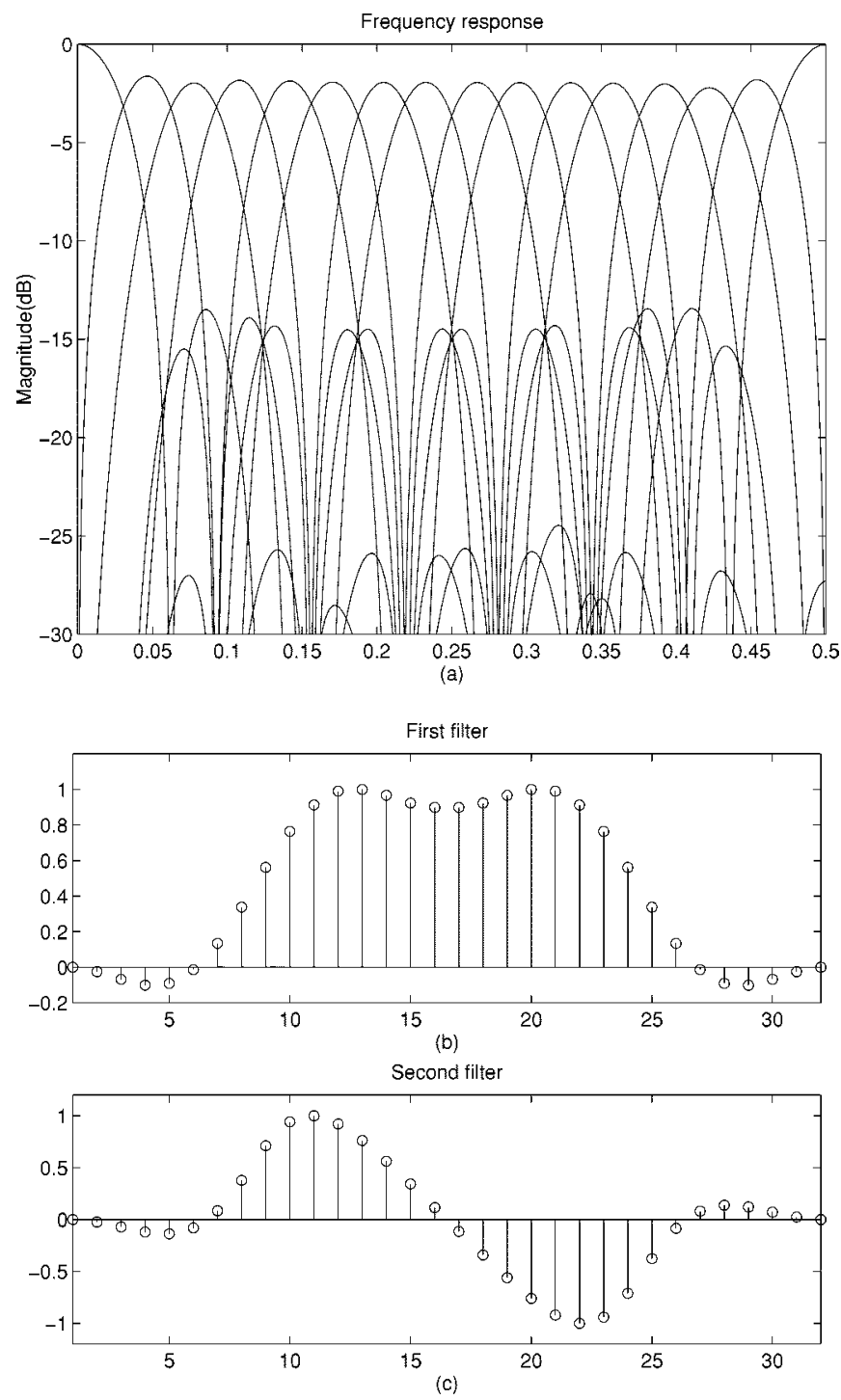

Fig. 4. Sixteen-channel LOT: (a) transfer functions, (b) first filter, and (c) second filter.

\section{Design ANd Coding EXAMPLes}

In this section, we present some design and coding examples. We demonstrate the tradeoff between decay of the filters in the time domain and frequency selectivity. Specifically, we show that LT-based coders achieve the same rate-distortion performance as DCT-based coders while visually being substantially better. The coder we used consists of a transform coding part, a uniform quantizer, and an entropy (Huffman) coder. In Fig. 3(a) the frequency responses of the filters in a 16-channel LOT are depicted. The filters have been obtained by minimization of $J_{1, k}$ in each step of the iteration (see Section IV-A). In Fig. 3(b) and (c), the impulse responses of the first and the second filter, respectively, are depicted. Clearly the filters are "smooth" in the time domain. Note that these filters will still lead to blocking effects, since their impulse responses do not decay to zero at the boundaries.

In Fig. 4 a 16-channel LOT obtained by minimizing $J_{2, k}$ in each step of the iteration is shown. The weighting function $W_{k}\left(e^{j 2 \pi \theta}\right)$ has been chosen as $W_{k}\left(e^{j 2 \pi \theta}\right)=\left(\theta-\theta_{k}\right)^{2}$. In this 


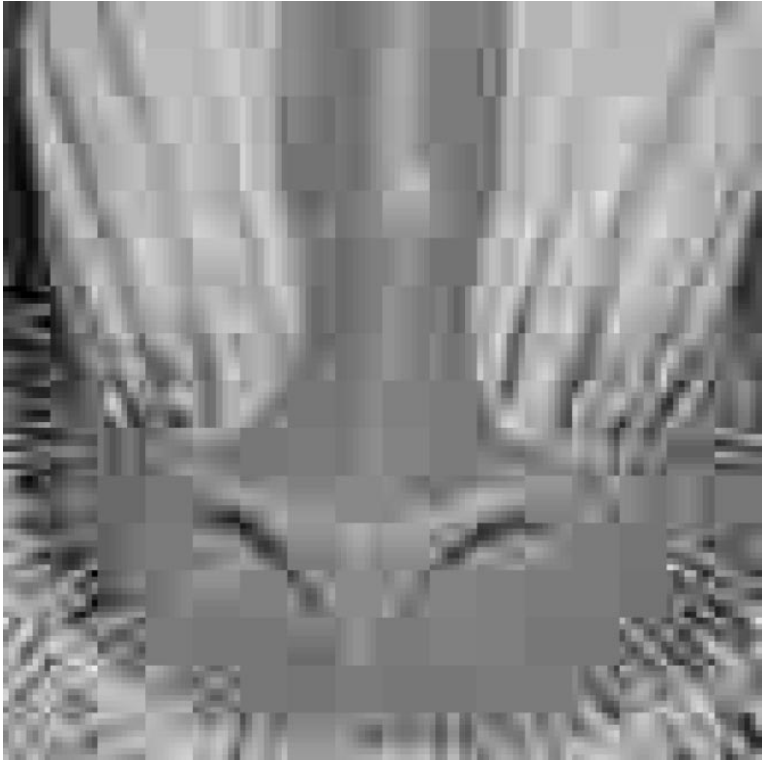

(a)

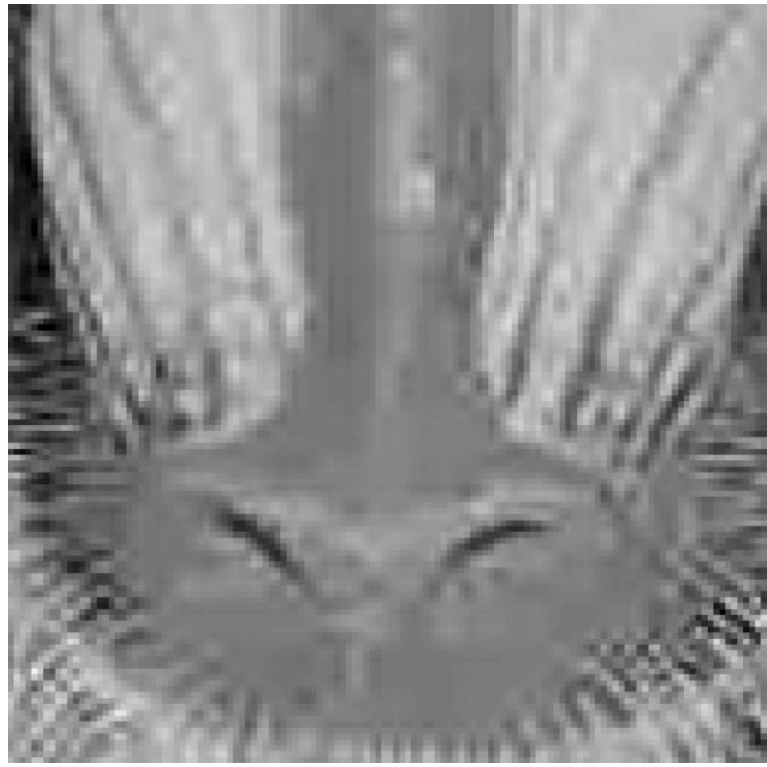

(b)

Fig. 5. A coding example: (a) "baboon" coded at $0.3 \mathrm{bpp}(\mathrm{PSNR}=24.54 \mathrm{~dB})$ using the DCT and (b) coded at $0.3 \mathrm{bpp}(\mathrm{PSNR}=24.63 \mathrm{~dB})$ using the LOT depicted in Fig. 6.

case the blocking effects will be completely eliminated and the filters are still "smooth." Note however, that the price one has to pay for the elimination of the blocking effects is a decrease in the stopband attenuation of the filters. This can be seen by comparing Figs. 3(a) and 4(a).

We shall next present a coding example ${ }^{9}$ that serves to demonstrate the perceptual performance of LOT's designed using our method as compared to the DCT. Part of the image "baboon" has been coded (the subband signals were Huffman coded) at a rate of 0.3 bpp. Fig. 5(a) shows the result of the DCT-based coder. In Fig. 5(b) the result of a LOT-based coder is depicted.

${ }^{9}$ The 2-D transform we used is a separable extension of the 1-D transform.

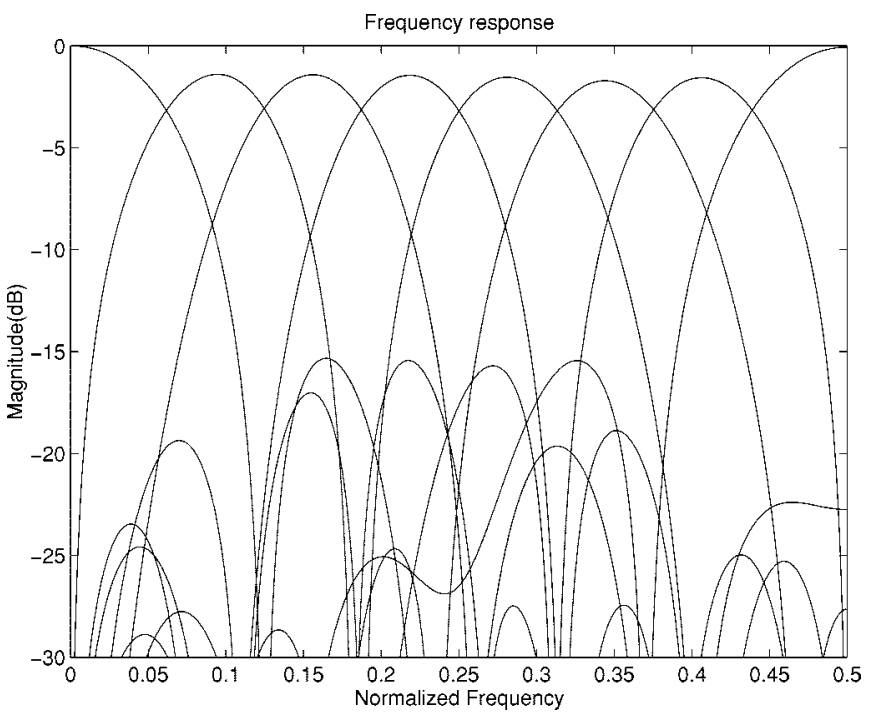

(a)

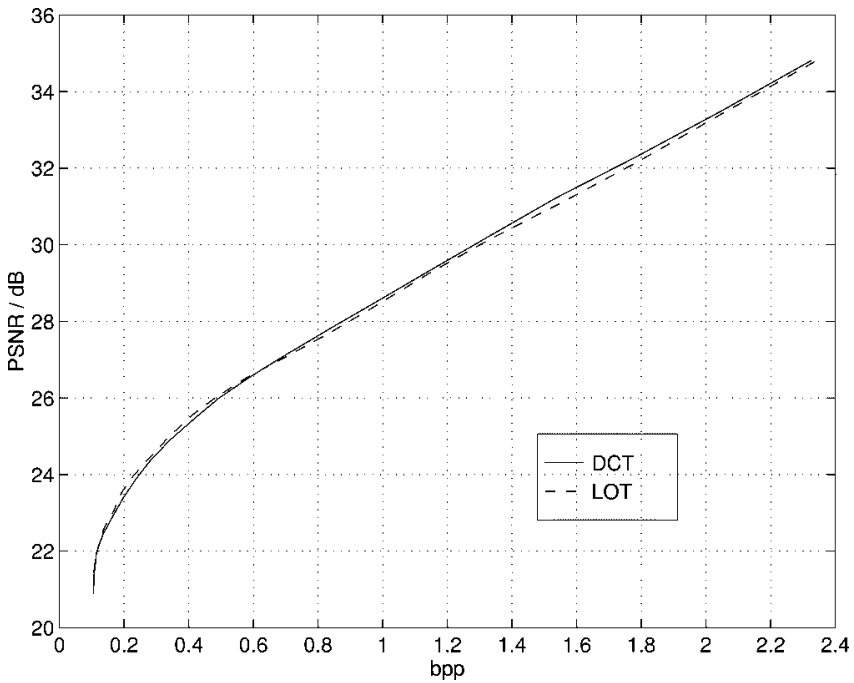

(b)

Fig. 6. (a) Transfer functions of the LOT used in the coding experiment in Fig. 5 and (b) comparison of the rate-distortion performance of DCT (blocksize $=$ 8) and the LOT depicted in (a).

The LOT transfer functions used in this coding example are shown in Fig. 6(a). Finally, Fig. 6(b) demonstrates that the LOTbased coder (transform coding plus Huffman coding) achieves the same error-rate performance as the DCT-based coder.

From the discussion in Section III we know that in order to avoid blocking effects, the synthesis filters should decay to zero at the ends of their supporting intervals. The analysis filters need not satisfy this condition. Therefore, it is of interest that only the synthesis filters decay to zero. In the orthogonal case this is not possible, since the analysis filters are equal to the synthesis filters (up to time reversal). In the biorthogonal case we can satisfy this requirement, since we can impose different restrictions on the analysis and the synthesis filters. The following design example presents a BOLT that has been designed using the procedure described in Section IV-C. The transform consists of eight basis functions each of length 16 . 

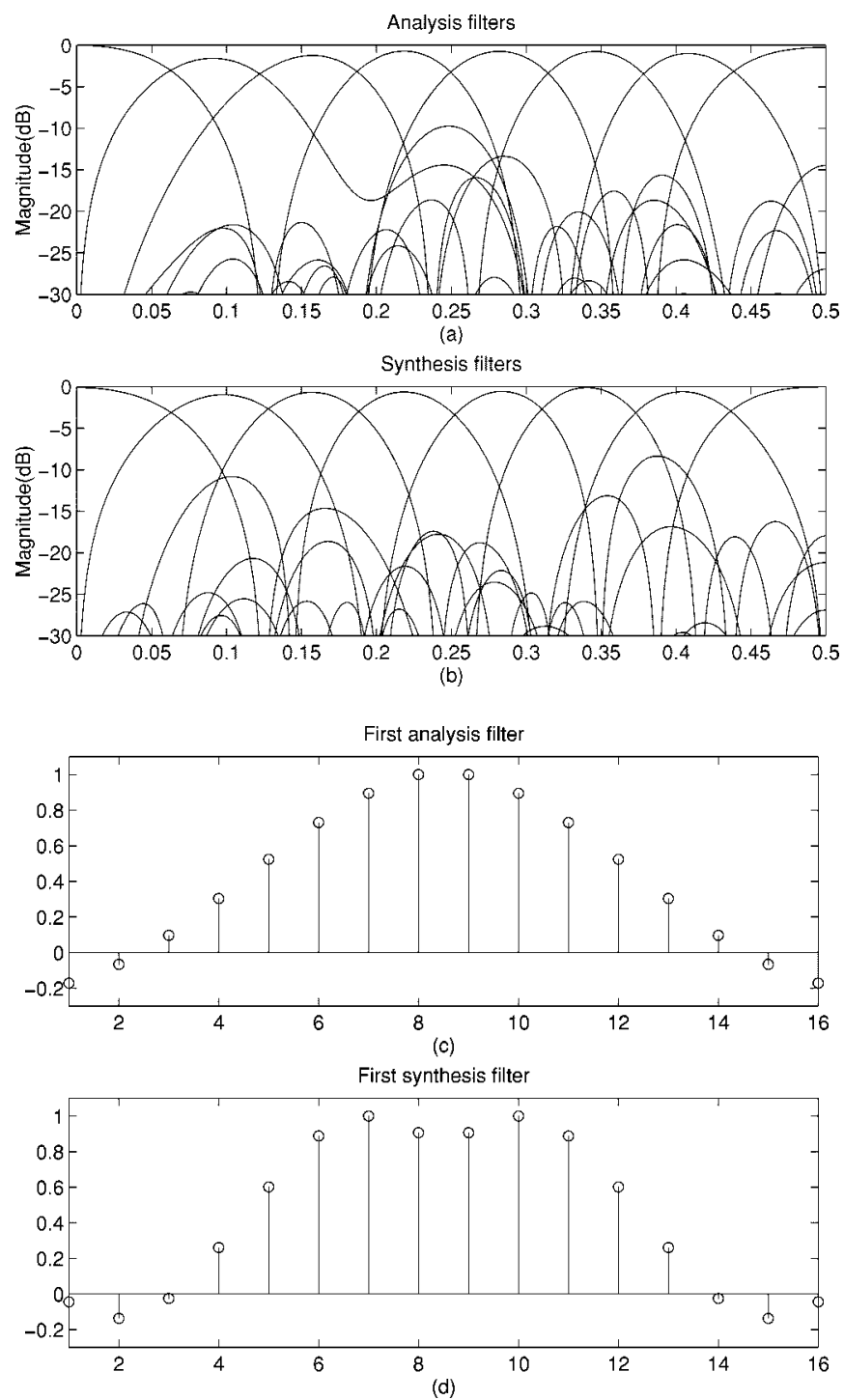

Fig. 7. Eight-channel BOLT: (a) transfer functions of analysis filters, (b) transfer functions of synthesis filters, (c) first analysis filter, and (d) first synthesis filter.

We used the cost functions $J_{2, k}$ defined in Section IV-C and modified them such that they contain the boundary values $f_{k}[0]$ of the filters. Thus, $J_{2, k}=\tilde{\mathbf{h}}_{k}^{T} \mathbf{S}_{k} \tilde{\mathbf{h}}_{k}+\lambda_{1}^{\prime} \tilde{\mathbf{f}}_{k}^{T} \mathbf{U}_{k} \tilde{\mathbf{f}}_{k}+\lambda_{2}^{\prime} f_{k}^{2}[0]$ with $k=0,1, \cdots, M / 2-1$. Consequently, the side constraint $f_{k}[0]=0$ has been omitted. The weighting factors were $\lambda_{1}^{\prime}=$ $6, \lambda_{2}^{\prime}=3$. Fig. 7(a) and (b) shows the transfer functions of the analysis and synthesis filters, respectively. Fig. 7(c) and (d) shows the first analysis filter and the first synthesis filter, respectively. It can be seen that the impulse response of the first synthesis filter decays to zero at its boundaries, whereas the corresponding analysis filters still exhibit discontinuities at its boundaries. The frame bounds of this transform are $A=0.696$ and $B=1.325(B / A=1.903)$, which implies good numerical properties or equivalently small noise amplification.

Finally, in order to demonstrate that our algorithms are capable of designing LT's with a large number of channels, we show in Fig. 8 a 64-channel LOT obtained by minimizing $J_{2, k}$ in each step of the iteration. The weighting function $W_{k}\left(e^{j 2 \pi \theta}\right)$ has been chosen as $W_{k}\left(e^{j 2 \pi \theta}\right)=\left(\theta-\theta_{k}\right)^{2}$.
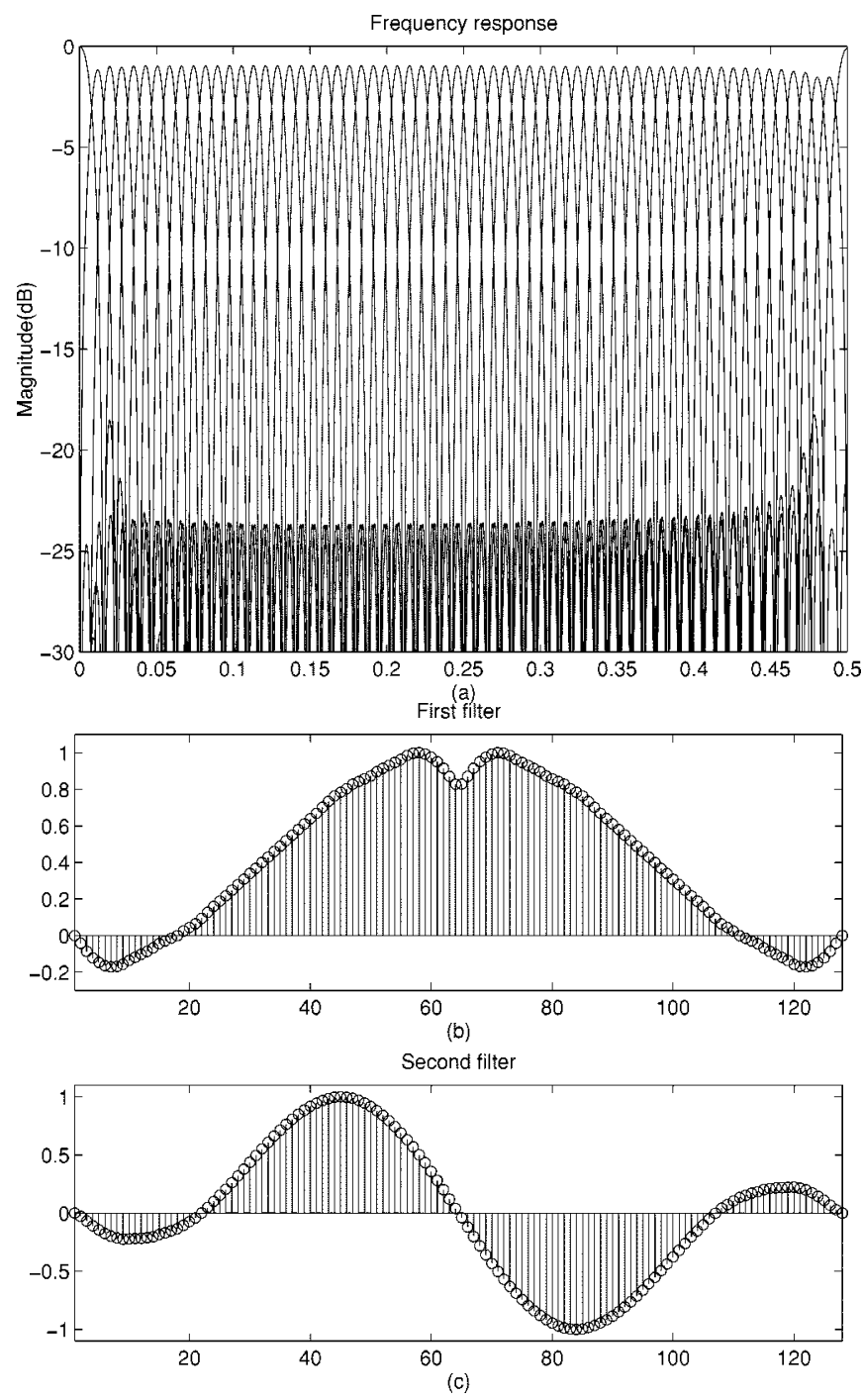

Fig. 8. Sixty-four channel LOT: (a) transfer functions, (b) first filter, and (c) second filter.

\section{CONCLUSION}

Based on a decomposition of LT's with 50\% overlap we formulated an efficient recursive method for the design of orthogonal and biorthogonal LT's for image coding applications. The proposed decompositon allows to decouple the design of the even-symmetric and the odd-symmetric filters, which drastically reduces the number of variables that have to be optimized at a time. It furthermore reveals all the variables that are predetermined due to perception related and coding-efficiency related constraints imposed on the filters. The proposed optimization procedure is robust with respect to initial solutions. It has been demonstrated that perceptually related constraints such as decay and smoothness of the filters' impulse responses can easily be incorporated in the optimization procedure. BOLT's offer more design flexibility than LOT's. We provided design examples and we demonstrated by means of a coding example that coders based on LT's designed using our approach achieve the same rate-distortion performance as DCT-based coders while visually performing substantially better. Finally, we demonstrated that 
our approach allows for the design of LT's with a large number of channels.

\section{ACKNOWLEDGMENT}

The hospitality of Philips Research Laboratories is gratefully acknowledged.

\section{REFERENCES}

[1] N. Ahmed, T. Natarajan, and K. R. Rao, "Discrete cosine transform," IEEE Trans. Comput., pp. 90-93, Jan. 1974.

[2] P. Cassereau, "A new class of optimal unitary transforms for image processing," M.S. thesis, Mass. Inst. Technol., Cambridge, MA, May 1985.

[3] H. S. Malvar and D. H. Staelin, "The LOT: Transform coding without blocking effects," IEEE Trans. Acoust., Speech, Signal Processing, vol. 37, pp. 553-559, Apr. 1989.

[4] H. S. Malvar, "Lapped transforms for efficient transform/subband coding," IEEE Trans. Acoust., Speech, Signal Processing, vol. 38, pp. 969-978, June 1990

[5] —_, "Modulated QMF filter banks with perfect reconstruction," Electron. Lett., vol. 26, pp. 906-907, June 1990.

[6] - Signal Processing with Lapped Transforms. Norwood, MA: Artech House, 1992.

[7] M. Vetterli and D. LeGall, "Perfect reconstruction FIR filter banks: Some properties and factorizations," IEEE Trans. Acoust., Speech, Signal Processing, vol. 37, pp. 1057-1071, July 1989.

[8] R. L. de Queiroz, T. Q. Nguyen, and K. R. Rao, "The GenLOT: Generalized linear-phase lapped orthogonal transform," IEEE Trans. Signal Processing, vol. 44, pp. 497-507, Mar. 1996.

[9] P. P. Vaidyanathan and T. Chen, "Role of anticausal inverses in multirate filter banks-Part II: The FIR case, factorizations, and biorthogonal lapped transforms," IEEE Trans. Signal Processing, vol. 43, pp. 1103-1115, May 1995.

[10] R. Heusdens, "Design of lapped orthogonal transforms," IEEE Trans. Image Processing, vol. 5, pp. 1281-1284, Aug. 1996.

[11] _ _ "Overlapped transform coding of images: Theory, application and realization," Ph.D. dissertation, Delft Univ. Technol., Delft, The Netherlands, Mar. 1997.

[12] P. P. Vaidyanathan, Multirate Systems and Filter Banks. Englewood Cliffs, NJ: Prentice-Hall, 1993.

[13] M. Vetterli and J. Kovačević, Wavelets and Subband Coding. Englewood Cliffs, NJ: Prentice-Hall, 1995.

[14] M. Vetterli, "A theory of multirate filter banks," IEEE Trans. Acoust., Speech, Signal Processing, vol. ASSP-35, pp. 356-372, Mar. 1987.

[15] P. P. Vaidyanathan, "Theory and design of $M$-channel maximally decimated quadrature mirror filters with arbitrary $M$, having perfect reconstruction property," IEEE Trans. Acoust., Speech, Signal Processing, vol. ASSP-35, pp. 476-492, Apr. 1987.

[16] H. Bölcskei, F. Hlawatsch, and H. G. Feichtinger, "Frame-theoretic analysis of oversampled filter banks," IEEE Trans. Signal Processing, vol. 46, pp. 3256-3268, Dec. 1998

[17] H. Bölcskei, "Oversampled filter banks and predicative subband coders," Ph.D. dissertation, Vienna Univ. Technol., Nov. 1997.

[18] H. Bölcskei, F. Hlawatsch, and H. G. Feichtinger, "Frame-theoretic analysis and design of oversampled filter banks," in Proc. IEEE ISCAS-96, vol. 2, Atlanta, GA, May 1996, pp. 409-412.

[19] D. J. Sakrison and V. R. Algazi, "Comparison of line-by-line and twodimensional encoding of random images," IEEE Trans. Inform. Theory, vol. 17 , pp. 386-398, July 1971.

[20] J. L. Mannos and D. J. Sakrison, "The effect of a visual fidelity criterion on the encoding of images," IEEE Trans. Inform. Theory, vol. IT-20, pp. 525-536, July 1974.

[21] A. N. Netravali and B. Prasada, "Adaptive quantization of picture signals using spatial masking," Proc. IEEE, vol. 65, pp. 536-548, Apr. 1977.

[22] A. K. Soman, P. P. Vaidyanathan, and T. Q. Nguyen, "Linear phase filter banks: Theory, factorizations and designs," IEEE Trans. Signal Processing, vol. 41, pp. 3480-3496, Dec. 1993.

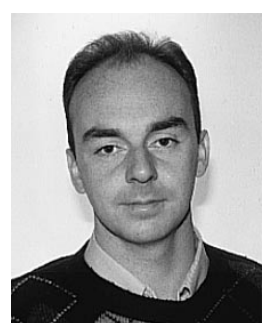

Helmut Bölcskei (S'94-M'98) was born in Austria on May 29, 1970. He received the Dipl.-Ing. and Dr.Tech. degrees from Vienna University of Technology, Vienna, Austria, in 1994 and 1997, respectively

From October to November 1994, he was a Researcher with the Department of Mathematics, University of Vienna. Since December 1994, he has been with the Department of Communications and RadioFrequency Engineering, Vienna University of Technology, where he is an Assistant Professor. From February to May 1996, he was a Visiting Researcher with the Applied Mathematics Group at Philips Research Laboratories Eindhoven, The Netherlands, where he worked on video coding. From February to March 1998, he visited ENST Paris, Paris, France, where he was involved in research on multicarrier modulation. Since February 1999, he has been on leave to do his post-doctoral work jointly in the Information Systems Laboratory and the Department of Statistics, Stanford University, Stanford, CA. His research interests include communication theory and signal processing with special emphasis on smart antennas, OFDM, and parameter estimation problems in the context of wireless communications.

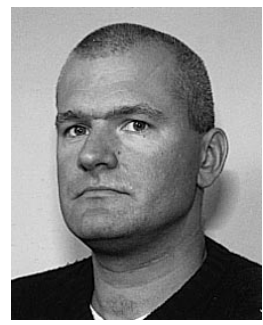

Richard Heusdens received the M.S. and Ph.D. degrees in electrical engineering from Delft University of Technology, Delft, The Netherlands, in 1992 and 1997, respectively.

In 1992, he joined the Digital Signal Processing Group, Philips Research Laboratories, Eindhoven, The Netherlands, where he worked mainly on advanced algorithms for audio/video compression. Since 1997, he has been an Associate Professor with the Delft University of Technology. His research interests include audio/video compression, VLSI architectures for signal transforms, and wireless communication.

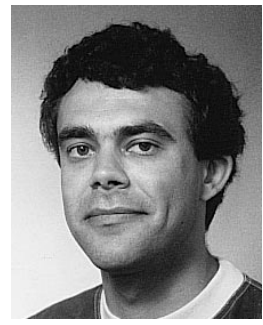

Rik Theunis was born in The Netherlands in 1965. He received the M.Sc. degree from the Department of Electrical Engineering, Delft University of Technology, Delft, The Netherlands, in 1992.

In 1992, he joined the Digital Signal Processing Group, Philips Research Laboratories, Eindhoven, The Netherlands. In 1997 he left Philips and founded Internet Multimedia Engineering, Velserbroek, The Netherlands.

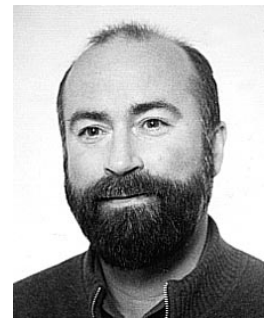

Augustus J. E. M. Janssen received the Eng. and $\mathrm{Ph} . \mathrm{D}$. degrees in mathematics from the Eindhoven University of Technology, Eindhoven, The Netherlands, in 1976 and 1979, respectively.

From 1979 to 1981, he was a Bateman Research Instructor with the Mathematics Department, California Institute of Technology, Pasadena. In 1981, he joined the Philips Research Laboratories, Eindhoven, where his principal responsibility is to provide high level mathematical service and consultancy in mathematical analysis. His research interest is in Fourier analysis with emphasis on time-frequency analysis, in particular Gabor analysis. His current research interests include the Fourier analysis of nonlinear devices such as quantizers. He has published 90 papers in the fields of signal analysis, mathematical analysis, Wigner distribution and Gabor analysis, information theory, and electron microscopy. He has also published 33 internal reports and holds four U.S. patents.

Dr. Janssen received the prize for the best contribution to the Mathematical Entertainments column of the Mathematical Intelligencer in 1987 and the EURASIP 1988 Award for the Best Paper of the Year in Signal Processing. 